\title{
Optimal threshold density in a stochastic resource management model with pulse intervention
}

\author{
Yi Tan ${ }^{\dagger}$ Lijuan Ning ${ }^{\dagger}$ Sanyi Tang ${ }^{\dagger *}$ Robert A. Cheke ${ }^{\S}$ \\ $\dagger$ School of Mathematics and Information Science, Shaanxi Normal University \\ Xi'an, 710062, P.R. China \\ $\S$ Natural Resources Institute, University of Greenwich at Medway, \\ Central Avenue, Chatham Maritime, Chatham, Kent, ME4 4TB, UK
}

*Corresponding author. E-mail: sytang@snnu.edu.cn 


\section{Abstract}

Human activities and agricultural practices are having huge impacts on the development of fishery and land resources through different ways. In order to model such systems that involve harvesting, an impulsive model of natural resources with a stochastic noise perturbation element is formulated to study the relationship between (a) the maximal expectation of biomass after harvesting or fishing events and (b) the minimal expectation of pest biomass and the number of times pesticide is applied. Using a detailed analytical treatment, time estimation and numerical demonstrations, we establish that the proposed mechanism is capable of maximizing fish populations at the end of a fishing season and minimizing pest numbers after a crop harvesting season once the intensity of the noise is relative small. Investigations of the effects of different parameters reveal that theoretical predictions from the new stochastic model accord with those from the deterministic case

\section{Recommendations for Resource Managers}

- Various measures can be implemented to manage natural resources, such as adjusting fishing quantity and intensity to maximize fish population.

- In the natural environment, population growth is inevitably affected by the environment noise. So it is important to understand noise effect to maintain sustainability of resources.

- Investigated methods are useful to converse resources and can be widely applied to control pests.

Keywords: Pulse perturbation; Stochastic logistic equation; Optimal threshold density; Fishing time; Maximal biomass expectation. 


\section{Introduction}

Sustainable exploitation of renewable resources and pest management have become important topics in theoretical biology (Clark, 1976; Szuwalski et al., 2016; Punt and Hilborn, 1997; Anderson, 2004). Many mathematical models have been established to investigate how to keep a balance between economic development and protection of renewable resources. The use of impulsive differential equations makes it possible to characterise the dynamic behaviour of both exploited fish populations and pest populations subject to control measures (Xiao et al., 2006; Zhang et al., 2003; Tang and Chen, 2004; Nundloll et al., 2010; Terry and Gourley, 2010). In reality, however, the existence of noise is inevitable and the introduction of environmental noise into models has attracted much interest (Bandyopadhyay et al., 2008; Liu and Mao, 1998; Schaffer et al., 1986; Hassani et al., 2010; Cao and Tang, 2016; Arnold et al., 1979; Castro-santis et al., 2016; Kloeden and Pearson, 1992; Wang et al., 2017; Cai et al., 2017). The crucial feature of the introduction of noise is to accurately reflect environmental effects and describe the internal cycles of the growth and decay of populations. An understanding and analysis of the dynamic relationships involved is required to develop suitable management methods to utilize renewable resources optimally.

Here we explore a feasible method to maximize stochastically fluctuating natural resources such as fish or pest populations. Although a fish population under exploitation by a fishery could be influenced by many factors, man's action is considered to be the main controlling agent. An important management objective is to obtain the maximum average fish population density at the end of a given harvesting season $[0, T]$. To do so, we propose a general control mechanism of stochastically fluctuating fish populations, which consists of two parts. One is to search for optimal threshold densities of a fish population for initiating fishing activities, the other is to estimate the time when the fish population density will attain these thresholds.

Time estimation in a Malthusian case is relatively easy to obtain and has been addressed in (Castro-santis et al., 2016). However to obtain a suitable estimation of fishing times in a logistic model with noise requires some more detailed analyses. Our investigations involve established methods for multi-impulsive harvesting and non-constant harvesting models. We have also tested our conclusions by numerical simulation, the predictions of which are in good agreement with numerical experiments.

Our method is a tentative study of fishery resources which can be extended to other renewable resources such as agricultural systems with pests controlled according to the principles of integrated pest management (IPM). This pest control strategy has been discussed for several years, and many researchers have tried to supplement or amend the IPM concept (Tang et al., 2013; Tang and Cheke, 2008; Tang et al., 2005). Multiple-species resource management model has been investigated in reference (Zhao et al., 2003), and the optimal impulse control problems for periodic Gompertz system and theta logistic growth equation have been addressed in literatures (Dong et al., 2007; Zhao and Tang, 2011). It has been argued that decisions about pest prevention and control measures should take into account economic, social and ecological benefits. The purpose of IPM is to minimize the number of pests by keeping them under a certain economic threshold instead of eradicating 
them, by the combination of multiple protection measures, in particular by chemical and biological controls. In this investigation we will use our models determine the optimal time within a planting season of crops that minimizes a pest's density at the end of the season.

The paper is organized as follows. We present main results of deterministic model and derive the stochastic model in section 2. In section 3, a theoretical analysis of solving optimal thresholds is carried out. At first, a one-pulse impulsive constant harvesting model is investigated and then this is expanded into a two-pulse impulsive constant harvesting model, followed by a more general case of a multi-pulse impulsive harvesting system. Then, an analytical demonstration of the time estimation will be given in section 4 and all of the theoretical predictions will be verified by numerical simulations. In the final section, the conclusions are summarized.

\section{The model}

In fishery resources management, one of the practical issues that researchers and managers care about most is how to maximize fish populations at the end of a harvesting season. If fish populations live in a constant environment with a limited food supply and under constant impulsive harvesting, they can be modeled by the following impulsive differential equation

$$
\begin{cases}\frac{\mathrm{d} N(t)}{\mathrm{d} t}=r N(t)\left(1-\frac{N(t)}{K}\right), & t \neq \tau_{i}, i=1, \cdots, n, \\ N\left(\tau_{i}^{+}\right)=N\left(\tau_{i}\right)-Q, & t=\tau_{i}, i=1, \cdots, n,\end{cases}
$$

where $N(t)$ is the number or density of fish in the population at time $t, r$ represents the intrinsic rate of increase of the fish population not subject to environmental constraints, $K$ refers to the environmental carrying capacity, $Q$ is the fixed quota harvested and $N\left(\tau_{i}\right)>$ $Q, \tau_{i}$ for $i=1, \cdots, n$ are the time series of when fishing occurs and $N\left(\tau_{i}^{+}\right), i=1, \cdots, n$ represents the number of fish after harvesting at time $\tau_{i}$ for $i=1, \cdots, n$.

Given a time, $T$, representing a season or period during which the control tactics can be implemented at times $\tau_{i}$ in a multi-impulsive harvesting model, the problem of how to obtain maximal fish populations at the end of time $T$ has been studied in (Xue et al., 2012). We summarize the main results in (Xue et al., 2012) below. The solution of Eq.(1) on $\left(\tau_{i}, t\right]$ and the optimal harvesting times $\tau_{i}^{M}$ are given by

$$
\begin{aligned}
N(t) & =\frac{N\left(\tau_{i}^{+}\right) e^{r\left(t-\tau_{i}\right)}}{1+N\left(\tau_{i}^{+}\right)\left(e^{r\left(t-\tau_{i}\right)}-1\right) / K}, & i=1, \cdots, n, \\
\tau_{i}^{M} & =\frac{1}{r} \ln \left(\frac{K-N_{0}}{N_{0}}\left(\frac{K+Q}{K-Q}\right)^{2 i-1}\right)<T, & i=1, \cdots, n,
\end{aligned}
$$

where $N_{0}$ is the initial value of the fish population and $\tau_{i}^{M}$ is the timing or timings that give the maximum yield of the fish. Note that model (1) can be also employed to study the optimal pest control measures (i.e. minimize the pest population) if $N(t)$ represents 
the pest population and $Q$ is the number of killed pests at time $\tau_{i}$.

In fact, the problem of solving optimal fishing times to get maximal fish populations is equivalent to the problem of finding optimal thresholds $N_{i}$, which will be reached at $\tau_{i}$ for $i=1, \cdots, n$ such that when the fish population increases to $N_{i}$, impulsive harvesting activities are carried out. So we propose a state-dependent feedback control model:

$$
\begin{cases}\mathrm{d} N(t)=r N(t)\left(1-\frac{N(t)}{K}\right) \mathrm{d} t, & N(t)<N_{i}, i=1, \cdots, n \\ N_{i}^{+}=N_{i}-Q, & N(t)=N_{i}, i=1, \cdots, n\end{cases}
$$

which allows us to investigate similar questions as those posed for model (1) when we introduce stochasticity into the growth of the fish population.

Noise impacted models are widely used in almost all disciplines of natural science such as biology, physics, chemistry, etc. (Sakthivel and Luo, 2009; Caraballo, 1990; Imhof and Walcher, 2005; Campillo and Lobry, 2012; Campillo et al., 2011) and are of interest here as we handle a logistic model driven by random noise. We wish to investigate whether there exist optimal fishing times analogous to those derived from the deterministic version model (1). We illustrate the effect of additive Gaussian white noise $B(t)$, with zero mean and the correlation function $\langle B(t), B(s)\rangle=\sigma \delta(t-s)$, where $\sigma$ is the variance or intensity of the noise, and $\delta(t)$ is a Dirac function. Then the modified system becomes (Zou et al., 2013; Lu and Ding, 2014; Liu and Wang, 2012)

$$
\begin{cases}\mathrm{d} N(t)=r N(t)\left(1-\frac{N(t)}{K}\right) \mathrm{d} t+\sigma N(t) \mathrm{d} B(t), & N(t)<N_{i}, i=1, \cdots, n \\ N_{i}^{+}=N_{i}-Q, & N(t)=N_{i}, i=1, \cdots, n\end{cases}
$$

Here, it is reasonable to define successive harvest times $\tau_{i}:=\inf \left\{t>0: N(t)=N_{i}\right\}$. The existence and uniqueness of the solution of Eq.(4) can be similarly discussed according to the basic methods shown in (Castro-santis et al., 2016; Ji et al., 2007; Mao, 2007). Moreover, if $r>\sigma^{2} / 2$ then system (4) is persistent provided that the fixed quota harvested satisfies $N_{i}>Q$ and $N_{i}<N(T)$ for all $i=1, \cdots, n$ (Castro-santis et al., 2016), and the latter inequality is equivalent to assuming that $\tau_{i}<T$ in deterministic case (Xue et al., 2012).

In this paper, our main aim is to obtain optimal fishing thresholds for fish populations with noise perturbation and investigate how the frequency of fishing times affects how the fish populations can be maximized. After investigating optimal thresholds to maximize the level of biomass, a theory of estimating the time when fish stocks will reach these optimal thresholds is put forward to gain a deeper understanding of the similarities and differences between a time-dependent model and a state-dependent model. 


\section{Maximal biomass}

\subsection{Single-pulse constant harvesting in a given interval $[0, T]$}

During a harvesting season $[0, T]$, the population size varies as impulsive harvesting is conducted at different times in the interval $[0, T]$. In this subsection, we pay attention to a less general case. Suppose that the harvesting quota is constant and that fish are only caught once during $[0, T]$, the model can then be reduced to

$$
\begin{cases}\mathrm{d} N(t)=r N(t)\left(1-\frac{N(t)}{K}\right) \mathrm{d} t+\sigma N(t) \mathrm{d} B(t), & N(t)<N_{1}, \\ N_{1}^{+}=N_{1}-Q, & N(t)=N_{1} .\end{cases}
$$

For any initial value $N(0)=N_{0}$, it is interesting to determine whether there exists an optimal fishing threshold $N_{1}$ which will be reached at random time $\tau_{1}$ to get the largest population or maximal biomass at $T$ in the presence of additive white noise when $N_{1}<N(T)$. The introduction of the random noise will make modeling the growth of the fish population more complicated. Since it is difficult to obtain explicit solutions of the stochastic model, we cannot obtain the exact optimal fishing threshold and so we switch targets to maximize the expectation of the population and the optimal threshold $N_{1}$ can be solved in expectation.

Theorem 3.1. Suppose that $\sigma_{1}^{2} \triangleq r(K+Q-2 \sqrt{K Q}) / K$ and $0 \leqslant \sigma^{2}<\sigma_{1}^{2}<2 r$, then the expectation of fish population will reach its maximum in one-pulse harvesting model (5) when fishing activity is implemented at $N_{1}=\frac{-\sigma^{2} K+K r+3 Q r+\sqrt{A}_{1}}{4 r}$.

Proof. The solutions of Eq.(5) are piecewise continuous and we study them stepwise. We first investigate the following initial value problem on the time interval $[0, t]$ :

$$
\mathrm{d} N(t)=r N(t)\left(1-\frac{N(t)}{K}\right) \mathrm{d} t+\sigma N(t) \mathrm{d} B(t), N(0)=N_{0}
$$

with $t<\tau_{1}$. Using the Itô formula (Mao, 2007; Mao et al., 2003) for $f(x)=\ln |x-Q|$, we obtain

$$
\mathrm{d} \ln |N(t)-Q|=\frac{r N(t)\left(1-\frac{N(t)}{K}\right)}{|N(t)-Q|} \mathrm{d} t-\frac{\sigma^{2} N(t)^{2}}{2(N(t)-Q)^{2}} \mathrm{~d} t+\frac{\sigma N(t)}{|N(t)-Q|} \mathrm{d} B(t) .
$$

Integrating the above equation from 0 to $\tau_{1}$, yields

$$
\begin{aligned}
\ln \left(N_{1}-Q\right)= & \ln \left|N_{0}-Q\right|+\int_{0}^{\tau_{1}} \frac{r N(t)\left(1-\frac{N(t)}{K}\right)}{|N(t)-Q|} d t \\
& -\int_{0}^{\tau_{1}} \frac{\sigma^{2} N(t)^{2}}{2(N(t)-Q)^{2}} d t+\int_{0}^{\tau_{1}} \frac{\sigma N(t)}{|N(t)-Q|} d B(t) .
\end{aligned}
$$


By taking expectation, the last term $\int_{0}^{\tau_{1}} \sigma N(t) /|N(t)-Q| d B(t)$ can be regarded as a noise term and vanishes to zero because the Gauss property of stochastic integral

$$
\mathbb{E}\left[\int_{0}^{\tau_{1}} \sigma N(t) /|N(t)-Q| d B(t)\right] \sim N\left(0, \int_{0}^{\tau_{1}}(\sigma N(t) /|N(t)-Q|)^{2} d t\right),
$$

the expectation of this term is zero (Yeh, 1995), i.e. $\mathbb{E}\left[\int_{0}^{\tau_{1}} \sigma N(t) /|N(t)-Q| d B(t)\right]=0$. Therefore, the expectation of Eq.(8) is

$\mathbb{E}\left[\ln \left(N_{1}-Q\right)\right]=\ln \left|N_{0}-Q\right|+\mathbb{E}\left[\int_{0}^{\tau_{1}} \frac{r N(t)\left(1-\frac{N(t)}{K}\right)}{|N(t)-Q|} d t\right]-\mathbb{E}\left[\int_{0}^{\tau_{1}} \frac{\sigma^{2} N(t)^{2}}{2(N(t)-Q)^{2}} d t\right]$.

Then we investigate the equation defined in the time interval $\left(\tau_{1}, t\right]$, i.e.

$$
\mathrm{d} N(t)=r N(t)\left(1-\frac{N(t)}{K}\right) \mathrm{d} t+\sigma N(t) \mathrm{d} B(t)
$$

with $t \leq T$ and initial value is $N_{1}^{+}$. Similarly, we use the Itô formula for $g(x)=\ln x$ and obtain

$$
\mathrm{d} \ln N(t)=r\left(1-\frac{N(t)}{K}\right) \mathrm{d} t-\frac{1}{2} \sigma^{2} \mathrm{~d} t+\sigma \mathrm{d} B(t)
$$

Taking the integral from $\tau_{1}$ to $T$, the solution of Eq.(11) yields the expression

$$
\ln N(T)=\ln N_{1}^{+}+\left(r-\frac{1}{2} \sigma^{2}\right)\left(T-\tau_{1}\right)-\frac{r}{K} \int_{\tau_{1}}^{T} N(t) d t+\int_{\tau_{1}}^{T} \sigma d B(t)
$$

We can also ignore the last noise term by taking expectation and obtain

$$
\left.\mathbb{E}[\ln N(T)]=\mathbb{E}\left[\ln N_{1}^{+}+\left(r-\frac{1}{2} \sigma^{2}\right)\left(T-\tau_{1}\right)\right]-\frac{r}{K} \int_{\tau_{1}}^{T} N(t) d t\right] .
$$

Substituting Eq.(10) into Eq.(14), yields

$$
\begin{aligned}
\mathbb{E}[\ln N(T)] & =\ln \left|N_{0}-Q\right|+\mathbb{E}\left[\int _ { 0 } ^ { \tau _ { 1 } } \left(\frac{r N(t)\left(1-\frac{N(t)}{K}\right)}{|N(t)-Q|}\right.\right. \\
& \left.\left.-\frac{\sigma^{2} N(t)^{2}}{2(N(t)-Q)^{2}}\right) d t\right]+\left(r-\frac{1}{2} \sigma^{2}\right)\left(T-\mathbb{E}\left[\tau_{1}\right]\right)-\mathbb{E}\left[\frac{r}{K} \int_{\tau_{1}}^{T} N(t) d t\right] .
\end{aligned}
$$


In order to use the methods of solving the extreme value to maximize the stock level at $T$, we seek the derivative of $\mathbb{E}[\ln N(T)]$ with respect to $\tau_{1}$,

$$
\frac{\mathrm{d} \mathbb{E}[\ln N(T)]}{\mathrm{d} \tau_{1}}=\mathbb{E}\left[\frac{r N_{1}\left(1-\frac{N_{1}}{K}\right)}{N_{1}-Q}-\frac{\sigma^{2} N_{1}^{2}}{2\left(N_{1}-Q\right)^{2}}\right]-\left(r-\frac{1}{2} \sigma^{2}\right)+\mathbb{E}\left[\frac{r}{K} N_{1}^{+}\right] .
$$

Note that $N_{1}$ is given as a predetermined value and its expectation is constant. Thus, it follows from $\frac{\mathrm{dE}[\ln N(T)]}{\mathrm{d} \tau_{1}}=0$ that we can consider the following quadratic equation:

$$
-4 r N_{1}^{2}+\left(6 Q r+2 K r-2 \sigma^{2} K\right) N_{1}+\sigma^{2} K Q-2 K Q r-2 Q^{2} r=0 .
$$

In order for the discriminant $A_{1} \triangleq \sigma^{4} K^{2}-2 \sigma^{2} K^{2} r-2 \sigma^{2} K Q r+K^{2} r^{2}-2 K Q r^{2}+$ $Q^{2} r^{2}$ to be positive, we have either $0 \leqslant \sigma^{2}<r(K+Q-2 \sqrt{K Q}) / K \triangleq \sigma_{1}^{2}$, or $\sigma^{2}>$ $r(K+Q+2 \sqrt{K Q}) / K \triangleq \sigma_{2}^{2}$. Thus, the two roots of Eq.(17) can be written as

$$
\left\{\begin{array}{l}
N_{1}^{M}=\frac{-\sigma^{2} K+K r+3 Q r+\sqrt{A}_{1}}{4 r}, \\
N_{1}^{m}=\frac{-\sigma^{2} K+K r+3 Q r-\sqrt{A}_{1}}{4 r} .
\end{array}\right.
$$

Note that if $N_{1}^{M} N_{1}^{m}=\left(2 K Q r+2 Q^{2} r-\sigma^{2} K Q\right) / 4 r \leqslant 0$, then we have $\sigma^{2} \geqslant(2 K r+$ $2 Q r) / K>\sigma_{2}^{2}$. However, it follows from $\sigma^{2}<2 r$ and $2 r<(2 K r+2 Q r) / K$ that it is impossible that Eq.(17) has a positive and a negative root. Therefore, in order to ensure that Eq.(17) exists two positive roots, we must have

$$
\left\{\begin{array}{l}
N_{1}^{M} N_{1}^{m}=\frac{2 K Q r+2 Q^{2} r-\sigma^{2} K Q}{4 r}>0 \\
N_{1}^{M}+N_{1}^{m}=\frac{6 Q r+2 K r-2 \sigma^{2} K}{4 r}>0
\end{array}\right.
$$

which need $\sigma^{2}<(K r+3 Q r) / K$. Thus, it follows from $\sigma_{1}^{2}<(K r+3 Q r) / K<\sigma_{2}^{2}$ that Eq.(17) has two positive roots provided that $0 \leqslant \sigma^{2}<\sigma_{1}^{2}<2 r$.

Hence, it is easy to know that the expectation of the population $N(T)$ will reach its maximum at

$$
N_{1}=N_{1}^{M}=\frac{-\sigma^{2} K+K r+3 Q r+\sqrt{A}_{1}}{4 r}
$$

provided that $0 \leqslant \sigma^{2}<\sigma_{1}^{2}<2 r$ and $N_{1}^{M}<N(T)$. All these results confirm that we should perform fishing activity once the fish population reaches thresholds such as $N_{1}^{M}$ so that in the subsequent growth, the expectation of the population will reach its maximum at time $T$. Note that the optimal threshold $N_{1}^{M}$ could only exist for a relatively small random perturbation.

To verify the validity of our theoretical calculations obtained in this subsection, we fix all parameters including $r, K, Q$ and $N_{0}$. Fig(1)(a) shows comparisons of fishing strategies between harvesting at the calculated optimal thresholds $N_{1}$ and at two other arbitrary 
setting values. Each of the trajectories is affected by noise and are simulated 40 times and then the averages are shown in $\operatorname{Fig}(1)(a)$. Trajectories of stochastically fluctuating fish populations without average are shown in $\operatorname{Fig}(1)(b)$ and $\operatorname{Fig}(1)(c)$. It is observed that only if we implement fishing activity at the calculated optimal thresholds $N_{1}$ can the expectation of the fish population be maximized at the end of the fishing season.

Remark 3.1. The solution of Eq.(3) for $i=1$ can be expressed as

$$
N(T)=N_{0}+\int_{0}^{\tau_{1}} r N(t)\left(1-\frac{N(t)}{K}\right) d t-Q+\int_{\tau_{1}}^{T} r N(t)\left(1-\frac{N(t)}{K}\right) d t .
$$

Taking the derivative of $N(T)$ with respect to $\tau_{1}$ yields

$$
\begin{aligned}
\frac{\mathrm{d} N(T)}{\mathrm{d} \tau_{1}} & =r N_{1}\left(1-\frac{N_{1}}{K}\right)-r N_{1}^{+}\left(1-\frac{N_{1}^{+}}{K}\right) \\
& =Q r+\frac{r Q^{2}}{K}-\frac{2 Q r}{K} N_{1} .
\end{aligned}
$$

Letting $\mathrm{d} N(T) / \mathrm{d} \tau_{1}=0$ and solving it with respect to $N_{1}$, we have $N_{1}^{M}=(K+Q) / 2$.

On the other hand, it follows from Eq.(2) with $i=1$ that the optimal fishing times

$$
\tau_{1}^{M}=\frac{1}{r} \ln \left(\frac{K-N_{0}}{N_{0}} \frac{K+Q}{K-Q}\right)
$$

which indicates that

$$
\begin{aligned}
N_{1}^{M} & =\frac{N_{0} e^{r \tau_{1}^{M}}}{1+N_{0}\left(e^{r \tau_{1}^{M}}-1\right) / K} \\
& =\frac{\left(K-N_{0}\right) \frac{K+Q}{K-Q}}{1+N_{0}\left(\frac{K-N_{0}}{N_{0}} \frac{K+Q}{K-Q}-1\right) / K} \\
& =\frac{K+Q}{2} .
\end{aligned}
$$

Furthermore, if we let $\sigma=0$ in Eq.(20), the optimal threshold in the deterministic model can again be obtained which is also $(K+Q) / 2$.

This result provides strong support for our conclusion that the stochastic model is equivalent to the deterministic model as the noise intensity $\sigma=0$, i.e. in the absence of noise.

Remark 3.2. The derivative of $N_{1}^{M}$ with respect to $\sigma$ yields

$$
\frac{\mathrm{d} N_{1}^{M}}{\mathrm{~d} \sigma}=\frac{-\sigma K\left(-\sigma^{2} K+K r+Q r+\sqrt{A_{1}}\right)}{2 r \sqrt{A_{1}}} .
$$

It follows from $\sigma^{2}<r(K+Q-2 \sqrt{K Q}) / K$ that we have

$$
-K \sigma^{2}+r K+r Q>2 r \sqrt{K Q} .
$$


It can be seen that $N_{1}^{M}$ is a monotonically decreasing function of $\sigma$. Let $\mathrm{d} N_{1}^{M} / \mathrm{d} \sigma=0$, we have $\sigma=0$ is the maximum point of $N_{1}^{M}$, which indicates that the optimal threshold of the stochastic model is always smaller than its corresponding deterministic model in the single-pulse impulsive harvesting model.

Remark 3.3. Note that if $0 \leqslant \sigma^{2}<\sigma_{1}^{2}<2 r$ and $N_{1}<N(T)$, then there exists another extreme point $N_{1}^{m}$, at which the expectation of the population will reach its minimum at time $T$, and this can be used for pest management. If so, $Q$ represents the number of pests killed by a single pesticide application at $\tau_{1}$. In particular, if the noise intensity $\sigma$ vanishes, then the extreme point $N_{1}^{m}$ is reduced to $Q$. This indicates that the pesticides should be applied at the beginning of the season if $N_{0} \geqslant Q$, while the control measures should be implemented once the pest population reaches $Q$ provided that $N_{0}<Q$ (Xue et al., 2012). In the rest of this paper, we only focus on the fishery resources management as the pest management problems can be discussed in the same ways.

\subsection{Two-pulse constant harvesting in a given interval}

In this subsection, we choose the same constant harvest quota $Q$, for fisheries management again, but this time consider catching fish twice during the given interval $[0, T]$. In this case, our primary task is to find the two thresholds $N_{1}<N(T)$ and $N_{2}<N(T)$, at which impulsive harvesting should be implemented to maximize the fish population $\mathbb{E}[\ln N(T)]$. As with the model discussed before, the two-pulse impulsive model can be described as follows:

$$
\begin{cases}\mathrm{d} N(t)=r N(t)\left(1-\frac{N(t)}{K}\right) \mathrm{d} t+\sigma N(t) \mathrm{d} B(t), & N(t)<N_{i}, i=1,2, \\ N_{i}^{+}=N_{i}-Q, & N(t)=N_{i}, i=1,2 .\end{cases}
$$

The solutions of Eq.(27) can be expressed by the Itô formula for three different functions in different time domains, i.e. $f(x)=\ln |x-2 Q|, g(x)=\ln |x-Q|$ and $h(x)=\ln (x)$, i.e. we have

$$
\begin{gathered}
\ln \left|N_{1}-2 Q\right|=\ln \left|N_{0}-2 Q\right|+\int_{0}^{\tau_{1}} \frac{r N(t)\left(1-\frac{N(t)}{K}\right)}{|N(t)-2 Q|} d t \\
-\int_{0}^{\tau_{1}} \frac{\sigma^{2} N(t)^{2}}{2(N(t)-2 Q)^{2}} d t+\int_{0}^{\tau_{1}} \frac{\sigma N(t)}{|N(t)-2 Q|} d B(t), \\
\begin{array}{r}
\ln \left(N_{2}-Q\right)=\ln \left|N_{1}^{+}-Q\right|+\int_{\tau_{1}}^{\tau_{2}} \frac{r N(t)\left(1-\frac{N(t)}{K}\right)}{|N(t)-Q|} d t \\
-\int_{\tau_{1}}^{\tau_{2}} \frac{\sigma^{2} N(t)^{2}}{2(N(t)-Q)^{2}} d t+\int_{\tau_{1}}^{\tau_{2}} \frac{\sigma N(t)}{|N(t)-Q|} d B(t), \\
\ln N(T)=\ln N_{2}^{+}+\left(r-\frac{1}{2} \sigma^{2}\right)\left(T-\tau_{2}\right)-\frac{r}{K} \int_{\tau_{2}}^{T} N(t) d t+\int_{\tau_{2}}^{T} \sigma d B(t),
\end{array}
\end{gathered}
$$


where $N_{1} \neq 2 Q, \ln \left|N_{1}^{+}-Q\right|=\ln \left|N_{1}-2 Q\right|$ and $\ln N_{2}^{+}=\ln \left(N_{2}-Q\right)$.

Substituting the first two equations into the third one, we have the expressions of $\ln N(T)$. After taking expectations of $\ln N(T)$ and ignoring the noise term, the objective function can be written as

$$
\begin{aligned}
\mathbb{E}[\ln N(T)] & =\ln \left|N_{0}-2 Q\right|+\mathbb{E}\left[\int_{0}^{\tau_{1}} \frac{r N(t)\left(1-\frac{N(t)}{K}\right)}{|N(t)-2 Q|} d t\right. \\
& \left.-\int_{0}^{\tau_{1}} \frac{\sigma^{2} N(t)^{2}}{2(N(t)-2 Q)^{2}} d t\right]+\mathbb{E}\left[\int_{\tau_{1}}^{\tau_{2}} \frac{r N(t)\left(1-\frac{N(t)}{K}\right)}{|N(t)-Q|} d t\right. \\
& \left.-\int_{\tau_{1}}^{\tau_{2}} \frac{\sigma^{2} N(t)^{2}}{2(N(t)-Q)^{2}} d t\right]+\left(r-\frac{1}{2} \sigma^{2}\right)\left(T-\mathbb{E}\left[\tau_{2}\right]\right)-\mathbb{E}\left[\frac{r}{K} \int_{\tau_{2}}^{T} N(t) d t\right]
\end{aligned}
$$

The first derivatives of $\mathbb{E}[\ln N(T)]$ with respect to $\tau_{1}$ and $\tau_{2}$ are

$$
\begin{aligned}
\frac{\partial \mathbb{E}[\ln N(T)]}{\partial \tau_{1}}=\mathbb{E} & {\left[\frac{r N_{1}\left(1-\frac{N_{1}}{K}\right)}{\left|N_{1}-2 Q\right|}-\frac{\sigma^{2} N_{1}^{2}}{2\left(N_{1}-2 Q\right)^{2}}\right] } \\
& -\mathbb{E}\left[\frac{r N_{1}^{+}\left(1-\frac{N_{1}^{+}}{K}\right)}{\left|N_{1}^{+}-Q\right|}-\frac{\sigma^{2}\left(N_{1}^{+}\right)^{2}}{2\left(N_{1}^{+}-Q\right)^{2}}\right], \\
\frac{\partial \mathbb{E}[\ln N(T)]}{\partial \tau_{2}}=\mathbb{E} & {\left[\frac{r N_{2}\left(1-\frac{N_{2}}{K}\right)}{N_{2}-Q}-\frac{\sigma^{2} N_{2}^{2}}{2\left(N_{2}-Q\right)^{2}}\right] } \\
& -\mathbb{E}\left[\left(r-\frac{1}{2} \sigma^{2}\right)-\frac{r}{K} N_{2}^{+}\right] .
\end{aligned}
$$

Letting $\partial \mathbb{E}[\ln N(T)] / \partial \tau_{1}=0$ and $\partial \mathbb{E}[\ln N(T)] / \partial \tau_{2}=0$, by employing the methods shown in the above we consider the following two cases. If $N_{1}>2 Q$, then it follows from $\partial \mathbb{E}[\ln N(T)] / \partial \tau_{1}=0$ that we obtain

$$
-4 r N_{1}^{2}+\left(10 Q r+2 K r-2 \sigma^{2} K\right) N_{1}+\sigma^{2} K Q-4 K Q r-4 Q^{2} r=0 .
$$

Denote its discriminant $B_{1} \triangleq \sigma^{4} K^{2}-2 \sigma^{2} K^{2} r-6 \sigma^{2} K Q r+K^{2} r^{2}-6 K Q r^{2}+9 Q^{2} r^{2}$, and we conclude that if $0 \leqslant \sigma^{2}<r(K+3 Q-2 \sqrt{3 K Q}) / K<2 r$, then Eq.(31) has two positive roots, denoted by $N_{1}^{M}$ and $N_{1}^{m}$. In combination with the equation $\partial \mathbb{E}[\ln N(T)] / \partial \tau_{2}=$ 0 whose roots can be written as $N_{2}^{M}$ and $N_{2}^{m}$, we obtain a pair of points $\left(N_{1}^{M}, N_{2}^{M}\right)$ 
maximized by the expectation of $N(T)$, i.e. we have

$$
\left\{\begin{array}{l}
N_{1}=N_{1}^{M}=\frac{-\sigma^{2} K+K r+5 Q r+\sqrt{B_{1}}}{4 r}, \\
N_{2}=N_{2}^{M}=\frac{-\sigma^{2} K+K r+3 Q r+\sqrt{A_{1}}}{4 r}
\end{array}\right.
$$

for $0 \leqslant \sigma^{2}<r(K+3 Q-2 \sqrt{3 K Q}) / K<2 r$.

Meanwhile, a pair of points $\left(N_{1}^{m}, N_{2}^{m}\right)$ minimized by the expectation of $N(T)$ is given as follows:

$$
\left\{\begin{array}{l}
N_{1}=N_{1}^{m}=\frac{-\sigma^{2} K+K r+5 Q r-\sqrt{B_{1}}}{4 r}, \\
N_{2}=N_{2}^{m}=\frac{-\sigma^{2} K+K r+3 Q r-\sqrt{A_{1}}}{4 r}
\end{array}\right.
$$

for $0 \leqslant \sigma^{2}<r(K+3 Q-2 \sqrt{3 K Q}) / K<2 r$.

If $N_{1}<2 Q$, we denote $B_{1}^{\prime} \triangleq \sigma^{4} K^{2}+2 \sigma^{2} K^{2} r+6 \sigma^{2} K Q r+K^{2} r^{2}-6 K Q r^{2}+9 Q^{2} r^{2}$. A pair of points $\left(N_{1}^{M}, N_{2}^{M}\right)$ maximized by the expectation of $N(T)$ is given as follows:

$$
\left\{\begin{array}{l}
N_{1}=N_{1}^{M}=\frac{\sigma^{2} K+K r+5 Q r-\sqrt{B_{1}^{\prime}}}{4 r} \\
N_{2}=N_{2}^{M}=\frac{-\sigma^{2} K+K r+3 Q r+\sqrt{A_{1}}}{4 r}
\end{array}\right.
$$

for $0 \leqslant \sigma^{2}<r(K+Q-2 \sqrt{K Q}) / K<2 r$, and a pair of points $\left(N_{1}^{m}, N_{2}^{m}\right)$ minimized by the expectation of $N(T)$ is given as follows:

$$
\left\{\begin{array}{l}
N_{1}=N_{1}^{m}=\frac{\sigma^{2} K+K r+5 Q r+\sqrt{B_{1}^{\prime}}}{4 r} \\
N_{2}=N_{2}^{m}=\frac{-\sigma^{2} K+K r+3 Q r-\sqrt{A_{1}}}{4 r}
\end{array}\right.
$$

for $0 \leqslant \sigma^{2}<r(K+Q-2 \sqrt{K Q}) / K<2 r$.

For further verification in this subsection, as depicted in $\operatorname{Fig}(2)(\mathrm{a})$ we present the average of stochastically fluctuating fish populations when impulsive harvesting is carried out if the fish population reaches $N_{1}^{M}$ and $N_{2}^{M}$ in the two-pulse model for $N_{1}<2 Q$. The other trajectories of the fish populations are of the control group when impulsive harvesting takes place at $\left(N_{1}^{\prime}, N_{2}^{\prime}\right)$ and $\left(N_{1}^{\prime \prime}, N_{2}^{\prime \prime}\right)$. Trajectories of stochastically fluctuating fish populations without average are shown in $\operatorname{Fig}(2)(b)$ and $\operatorname{Fig}(2)(c)$. It is observed that only if we implement fishing activity at the calculated optimal thresholds $N_{1}^{M}$ and $N_{2}^{M}$ can the expectation of the fish population be maximized at the end of the fishing season.

Remark 3.4. In the deterministic model, if we let $i=2$ in Eq.(2), then the optimal fishing times are given as

$$
\left\{\begin{array}{l}
\tau_{1}^{M}=\frac{1}{r} \ln \left(\frac{K-N_{0}}{N_{0}} \frac{K+Q}{K-Q}\right), \\
\tau_{2}^{M}=\frac{1}{r} \ln \left(\frac{K-N_{0}}{N_{0}}\left(\frac{K+Q}{K-Q}\right)^{3}\right),
\end{array}\right.
$$


substituting them into the solution of $N(t)$ yields

$$
\left\{\begin{array}{l}
N_{1}^{M}=\frac{N_{0} e^{r \tau_{1}^{M}}}{1+N_{0}\left(e^{r \tau_{1}^{M}}-1\right) / K}=\frac{K+Q}{2} \\
N_{2}^{M}=\frac{N\left(\tau_{1}^{+}\right) e^{r\left(\tau_{2}^{M}-\tau_{1}^{M}\right)}}{1+N\left(\tau_{1}^{+}\right)\left(e^{r\left(\tau_{2}^{M}-\tau_{1}^{M}\right)}-1\right) / K}=\frac{K+Q}{2} .
\end{array}\right.
$$

Note that only if $K=3 Q$ then the optimal solution $N_{1}$ is $2 Q$, so we can ignore the special case $N_{1}=2 Q$ in Eq.(28).

On the other hand, if $N_{1}>2 Q$ and letting $\sigma=0$ in Eq.(32), we have

$$
\left\{\begin{array}{l}
N_{1}^{M}=\frac{K r+5 Q r+|K-3 Q| r}{4 r}, \\
N_{2}^{M}=\frac{K+Q}{2} .
\end{array}\right.
$$

Note that if the inequality $2 Q<N_{1}<K<3 Q$ holds, then $N_{1}^{M}=2 Q$ which is impossible due to $N_{1}>2 Q$. Hence, we have $N_{1}^{M}=N_{2}^{M}=(K+Q) / 2$ for $2 Q<N_{1}<3 Q \leqslant K$ and $2 Q<3 Q \leqslant N_{1}<K$.

If $N_{1}<2 Q$ and letting $\sigma=0$ in Eq.(34), the optimal thresholds in the deterministic model are

$$
\left\{\begin{array}{l}
N_{1}=\frac{K r+5 Q r-|K-3 Q| r}{4 r} \\
N_{2}=\frac{K+Q}{2}
\end{array}\right.
$$

Again, for $N_{1}<2 Q<3 Q<K$ the optimal threshold $N_{1}^{M}$ cannot be well defined due to $N_{1}<2 Q$. Thus, we have $N_{1}^{M}=N_{2}^{M}=(K+Q) / 2$ for $N_{1}<2 Q<K \leqslant 3 Q$ and $N_{1}<K<2 Q<3 Q$. Again, our main results confirm that the optimal thresholds of the stochastic model are equivalent to those of the deterministic model as the noise intensity $\sigma=0$ (Xue et al., 2012).

Remark 3.5. The derivative of $N_{1}^{M}$ in Eq.(32) in two-pulse harvesting model with respect to $\sigma$ yields

$$
\frac{\mathrm{d} N_{1}^{M}}{\mathrm{~d} \sigma}=\frac{-\sigma K\left(-\sigma^{2} K+K r+3 Q r+\sqrt{B_{1}}\right)}{2 r \sqrt{B_{1}}} .
$$

It follows from $\sigma^{2}<r(K+3 Q-2 \sqrt{3 K Q}) / K$ that we have $-K \sigma^{2}+r K+3 r Q>$ $2 r \sqrt{3 K Q}$, which indicates that $N_{1}^{M}$ in this case is a monotonically decreasing function of $\sigma$.

Meanwhile, taking the derivative of $N_{1}^{M}$ in Eq.(34) with respect to $\sigma$ yields

$$
\frac{\mathrm{d} N_{1}^{M}}{\mathrm{~d} \sigma}=\frac{-\sigma K\left(\sigma^{2} K+K r+3 Q r-\sqrt{B_{1}^{\prime}}\right)}{2 r \sqrt{B_{1}^{\prime}}}<0 .
$$

In combination with $\mathrm{d} N_{2}^{M} / \mathrm{d} \sigma<0$ we can conclude that the optimal thresholds of the stochastic model are always smaller than those of the corresponding deterministic model. 


\subsection{Multi-impulse constant harvesting}

In this subsection, we extend our conclusions to the multi-pulse constant harvesting case. Supposing that the fixed fishing quota is $Q$, our main task is to determine whether the fishing thresholds $N_{i}<N(T)$, for $i=1, \cdots, n$ exist to maximize the expectation of the fish population at the end of a given harvest season $[0, T]$. Solutions of multi-impulse Eq.(4) can be given piecewise as follows:

$$
\begin{gathered}
\ln \left|N_{i}-(n-i+1) Q\right|=\ln \left|N_{i-1}^{+}-(n-i+1) Q\right| \\
\quad+\int_{\tau_{i-1}}^{\tau_{i}} \frac{r N(t)\left(1-\frac{N(t)}{K}\right)}{|N(t)-(n-i+1) Q|} d t \\
\quad-\int_{\tau_{i-1}}^{\tau_{i}} \frac{\sigma^{2} N(t)^{2}}{2(N(t)-(n-i+1) Q)^{2}} d t \\
\quad+\int_{\tau_{i-1}}^{\tau_{i}} \frac{\sigma N(t)}{|N(t)-(n-i+1) Q|} d B(t), i=1, \cdots, n, \\
\ln N(T)=\ln N_{n}^{+}+\left(r-\frac{1}{2} \sigma^{2}\right)\left(T-\tau_{n}\right)-\frac{r}{K} \int_{\tau_{n}}^{T} N(t) d t+\int_{\tau_{n}}^{T} \sigma d B(t),
\end{gathered}
$$

where $N_{i} \neq(n-i+1) Q(i=1, \cdots, n-1)$ with $N_{0}^{+}=N_{0}, \tau_{0}=0$ and $\ln \left(N_{i}^{+}-(n-i) Q\right)=$ $\ln \left(N_{i}-(n-i+1) Q\right)$.

Substituting the first $n$ equations one by one into the last equation and taking the expectations, we obtain the expressions of the fish population $\mathbb{E}[\ln N(T)]$ :

$$
\begin{aligned}
\mathbb{E}[\ln N(T)]= & \ln \left|N_{0}-n Q\right|+\mathbb{E}\left[\sum_{i=1}^{n}\left(\int_{\tau_{i-1}}^{\tau_{i}} \frac{r N(t)\left(1-\frac{N(t)}{K}\right)}{|N(t)-(n-i+1) Q|} d t\right]\right] \\
& -\mathbb{E}\left[\sum_{i=1}^{n}\left(\int_{\tau_{i-1}}^{\tau_{i}} \frac{\sigma^{2} N(t)^{2}}{2(N(t)-(n-i+1) Q)^{2}} d t\right)\right] \\
& +\left(r-\frac{1}{2} \sigma^{2}\right)\left(T-\mathbb{E}\left[\tau_{n}\right]\right)-\mathbb{E}\left[\frac{r}{K} \int_{\tau_{n}}^{T} N(t) d t\right] .
\end{aligned}
$$


Furthermore, we seek the derivatives of $\mathbb{E}[\ln N(T)]$ with respect to $\tau_{1}, \tau_{2}, \cdots, \tau_{n}$

$$
\begin{aligned}
& \frac{\partial \mathbb{E}[\ln N(T)]}{\partial \tau_{i}}=\mathbb{E}\left[\frac{r N_{i}\left(1-\frac{N_{i}}{K}\right)}{\left|N_{i}-(n-i+1) Q\right|}-\frac{\sigma^{2} N_{i}^{2}}{2\left(N_{i}-(n-i+1) Q\right)^{2}}\right] \\
& -\mathbb{E}\left[\frac{r N_{i}^{+}\left(1-\frac{N_{i}^{+}}{K}\right)}{\left|N_{i}^{+}-(n-i) Q\right|}-\frac{\sigma^{2}\left(N_{i}^{+}\right)^{2}}{2\left(N_{i}^{+}-(n-i) Q\right)^{2}}\right], i=1, \cdots, n-1, \\
& \frac{\partial \mathbb{E}[\ln N(T)]}{\partial \tau_{n}}=\mathbb{E}\left[\frac{r N_{n}\left(1-\frac{N_{n}}{K}\right)}{N_{n}-Q}-\frac{\sigma^{2} N_{n}^{2}}{2\left(N_{n}-Q\right)^{2}}\right]-\left(r-\frac{1}{2} \sigma^{2}\right)+\mathbb{E}\left[\frac{r}{K} N_{n}^{+}\right] .
\end{aligned}
$$

Letting $\partial \mathbb{E}[\ln N(T)] / \partial \tau_{i}=0$ and denoting

$$
\begin{aligned}
C_{i} \triangleq & -2(2 n-2 i+1) \sigma^{2} K Q r+K^{2} r^{2}-2(2 n-2 i+1) K Q r^{2} \\
& +(2 n-2 i+1)^{2} Q^{2} r^{2}+\sigma^{4} K^{2}-2 \sigma^{2} K^{2} r
\end{aligned}
$$

then we only focus on the following two cases. If $N_{i}-(n-i+1) Q>0$ for $i=1, \cdots, n$, then the discriminants $C_{i}$ are positive. Therefore, the optimal thresholds which maximize the expectation of $N(T)$ can be solved as

$$
N_{i}=N_{i}^{M}=\frac{-\sigma^{2} K+K r+(2(n-i+1)+1) Q r+\sqrt{C_{i}}}{4 r}
$$

and the optimal thresholds which minimize the expectation of $N(T)$ are given as

$$
N_{i}=N_{i}^{m}=\frac{-\sigma^{2} K+K r+(2(n-i+1)+1) Q r-\sqrt{C_{i}}}{4 r}
$$

for $0 \leqslant \sigma^{2}<r(K+(2 n-2 i+1) Q-2 \sqrt{(2 n-2 i+1) K Q}) / K<2 r$.

If $N_{i}-(n-i+1) Q<0$, we denote the discriminants

$$
\begin{aligned}
C_{i}^{\prime} \triangleq & 2(2 n-2 i+1) \sigma^{2} K Q r+K^{2} r^{2}-2(2 n-2 i+1) K Q r^{2} \\
& +(2 n-2 i+1)^{2} Q^{2} r^{2}+\sigma^{4} K^{2}+2 \sigma^{2} K^{2} r, \quad i \neq n .
\end{aligned}
$$

Thus, the optimal thresholds which maximize the expectation of $N(T)$ can be solved as

$$
\left\{\begin{array}{l}
N_{i}=N_{i}^{M}=\frac{\sigma^{2} K+K r+(2(n-i+1)+1) Q r-\sqrt{C_{i}^{\prime}}}{4 r}, i \neq n, \\
N_{n}=N_{n}^{M}=\frac{-\sigma^{2} K+K r+3 Q r+\sqrt{C_{n}}}{4 r}
\end{array}\right.
$$


and the optimal thresholds which minimize the expectation of $N(T)$ are given as

$$
\left\{\begin{array}{l}
N_{i}=N_{i}^{m}=\frac{\sigma^{2} K+K r+(2(n-i+1)+1) Q r+\sqrt{C_{i}^{\prime}}}{4 r}, i \neq n \\
N_{n}=N_{n}^{m}=\frac{-\sigma^{2} K+K r+3 Q r-\sqrt{C_{n}}}{4 r}
\end{array}\right.
$$

for $0 \leqslant \sigma^{2}<r(K+Q-2 \sqrt{K Q}) / K<2 r$.

Note that there are many cases for the sign of $N_{i}-(n-i+1) Q$, which can be similarly discussed, so we omitted them here. If we employ the analytic strategies in three- and fourpulse harvesting model for $N_{i}<(n-i+1) Q$, numerical results are shown in Fig(3) and Fig(4). According to the average and non-average curves, the conclusions are verified that the expectation of the stock levels will be maximized if harvesting activities are carried out at calculated thresholds.

Remark 3.6. In the same way that we analyzed the one- and two-pulse harvesting models, we find that the level of biomass in these optimal times are all $(K+Q) / 2$. Moreover, if $\sigma=0$ in Eq.(46), we obtain optimal thresholds

$$
\left\{\begin{array}{l}
N_{i}^{M}=\frac{K+Q}{\frac{K}{2} Q}, i \neq n \\
N_{n}^{M}=\frac{K}{2}
\end{array}\right.
$$

for $(n-i+1) Q<N_{i}<(2 n-2 i+1) Q \leqslant K$ and $(n-i+1) Q<(2 n-2 i+1) Q \leqslant N_{i}<K$, $i \neq n$.

If $\sigma=0$ in Eq.(49), we have

$$
\left\{\begin{array}{l}
N_{i}^{M}=\frac{K+Q}{2}, i \neq n \\
N_{n}^{M}=\frac{K+Q}{2}
\end{array}\right.
$$

for $N_{i}<(n-i+1) Q<K \leqslant(2 n-2 i+1) Q$ and $N_{i}<K<(n-i+1) Q<(2 n-2 i+1) Q$, $i \neq n$.

Comparing the results of the multi-impulsive stochastic model by letting $\sigma=0$ we verify that our results are consistent with the deterministic model.

Remark 3.7. Taking the derivative of $N_{i}^{M}(i=1, \cdots, n)$ in Eq.(46) with respect to $\sigma$ yields

$$
\frac{\mathrm{d} N_{i}^{M}}{\mathrm{~d} \sigma}=\frac{-\sigma K\left(-\sigma^{2} K+K r+(2 n-2 i+1) Q r+\sqrt{C_{i}}\right)}{2 r \sqrt{C_{i}}} .
$$

It follows from $\sigma^{2}<r(K+(2 n-2 i+1) Q-2 \sqrt{(2 n-2 i+1) K Q}) / K$ that

$$
-K \sigma^{2}+r K+(2 n-2 i+1) r Q>2 r \sqrt{(2 n-2 i+1) K Q}
$$

which means that $N_{i}^{M}$ in this case is a monotonically decreasing function of $\sigma$. 
Meanwhile, taking the derivative of $N_{i}^{M}$ in Eq.(49) with respect to $\sigma$ yields

$$
\frac{\mathrm{d} N_{i}^{M}}{\mathrm{~d} \sigma}=\frac{-\sigma K\left(\sigma^{2} K+K r+(2 n-2 i+1) Q r-\sqrt{C_{i}^{\prime}}\right)}{2 r \sqrt{C_{i}^{\prime}}}<0, i \neq n .
$$

All those results further confirm that the optimal thresholds of the stochastic model are always smaller than the corresponding deterministic model.

\subsection{Multi-pulse impulsive harvesting with different fishing quotas}

In reality, it is unlikely that the numbers of fish harvest quotas are always equal, which requires further investigation. A multi-pulse non-constant harvesting model can be written as follows:

$$
\begin{cases}\mathrm{d} N(t)=r N(t)\left(1-\frac{N(t)}{K}\right) \mathrm{d} t+\sigma N(t) \mathrm{d} B(t), & N(t)<N_{i}, i=1, \cdots, n, \\ N_{i}^{+}=N_{i}-Q_{i}, & N(t)=N_{i}, i=1, \cdots, n,\end{cases}
$$

where $Q_{i}\left(<N_{i}\right)$ for $i=1, \cdots, n$ are different catches. In this section, we try to demonstrate that there are fishing thresholds $N_{i}(<N(T))$ for $i=1, \cdots, n$ to get a maximal expectation of the fish population at $T$. Solutions of Eq.(56) can be expressed piecewise by the Itô formula

$$
\begin{aligned}
\ln \left|N_{i}-\sum_{k=i}^{n} Q_{k}\right| & =\ln \left|N_{i-1}^{+}-\sum_{k=i}^{n} Q_{k}\right| \\
& +\int_{\tau_{i-1}}^{\tau_{i}} \frac{r N(t)\left(1-\frac{N(t)}{K}\right)}{\left|N(t)-\sum_{k=i}^{n} Q_{k}\right|} d t-\int_{\tau_{i-1}}^{\tau_{i}} \frac{\sigma^{2} N(t)^{2}}{2\left(N(t)-\sum_{k=i}^{n} Q_{k}\right)^{2}} d t \\
& +\int_{\tau_{i-1}}^{\tau_{i}} \frac{\sigma N(t)}{\left|N(t)-\sum_{k=i}^{n} Q_{k}\right|} d B(t), \quad i=1, \cdots, n, \\
\ln N(T)=\ln N_{n}^{+} & +\left(r-\frac{1}{2} \sigma^{2}\right)\left(T-\tau_{n}\right)-\frac{r}{K} \int_{\tau_{n}}^{T} N(t) d t+\int_{\tau_{n}}^{T} \sigma d B(t),
\end{aligned}
$$

where $N_{i} \neq \sum_{k=i}^{n} Q_{k}, N_{0}^{+}=N_{0}, \tau_{0}=0, \ln \left|N_{i}^{+}-\sum_{k=i+1}^{n} Q_{k}\right|=\ln \left|N_{i}-\sum_{k=i}^{n} Q_{k}\right|$, for $i=$ $1, \cdots, n-1$ and $\ln N_{n}^{+}=\ln \left(N_{n}-Q_{n}\right)$. 
The expectation of the fish population is

$$
\begin{aligned}
\mathbb{E}[\ln N(T)]= & \ln \left|N_{0}-\sum_{i=1}^{n} Q_{i}\right|+\mathbb{E}\left[\sum_{i=1}^{n}\left(\int_{\tau_{i-1}}^{\tau_{i}} \frac{r N(t)\left(1-\frac{N(t)}{K}\right)}{\left|N(t)-\sum_{k=i}^{n} Q_{k}\right|} d t\right]-\mathbb{E}\left[\sum _ { i = 1 } ^ { n } \left[\int_{\tau_{i-1}}^{\tau_{i}}\right.\right.\right. \\
& \left.\frac{\sigma^{2} N(t)^{2}}{2\left(N(t)-\sum_{k=i}^{n} Q_{k}\right)^{2}} d t\right]+\left(r-\frac{1}{2} \sigma^{2}\right)\left(T-\mathbb{E}\left[\tau_{n}\right]\right)-\mathbb{E}\left[\frac{r}{K} \int_{\tau_{n}}^{T} N(t) d t\right] .
\end{aligned}
$$

The first derivatives of $\mathbb{E}[\ln N(T)]$ with respect to $\tau_{i}$ for $i=1, \cdots, n$ yield

$$
\begin{aligned}
& \frac{\partial \mathbb{E}[N(T)]}{\partial \tau_{i}}=\mathbb{E}\left[\frac{r N_{i}\left(1-\frac{N_{i}}{K}\right)}{\left|N_{i}-\sum_{k=i}^{n} Q_{k}\right|}-\frac{\sigma^{2} N_{i}^{2}}{2\left(N_{i}-\sum_{k=i}^{n} Q_{k}\right)^{2}}\right] \\
& -\mathbb{E}\left[\frac{r N_{i}^{+}\left(1-\frac{N_{i}^{+}}{K}\right)}{\left|N_{i}^{+}-\sum_{k=i+1}^{n} Q_{k}\right|}-\frac{\sigma^{2}\left(N_{i}^{+}\right)^{2}}{2\left(N_{i}^{+}-\sum_{k=i+1}^{n} Q_{k}\right)^{2}}\right], i=1 \cdots, n-1, \\
& \frac{\partial \mathbb{E}[N(T)]}{\partial \tau_{n}}=\mathbb{E}\left[\frac{N_{n}\left(1-\frac{N_{n}}{K}\right)}{N_{n}-Q_{n}}-\frac{\sigma^{2} N_{n}^{2}}{2\left(N_{n}-Q_{n}\right)^{2}}\right]-\left(r-\frac{1}{2} \sigma^{2}\right)+\mathbb{E}\left[\frac{r}{K} N_{n}^{+}\right] .
\end{aligned}
$$

Let $\partial \mathbb{E}[\ln N(T)] / \partial \tau_{i}=0$ for $i=1, \cdots, n$ to solve the optimal threshold points $N_{i}$ for $i=1, \cdots, n$. The process is the same as for the previous proof used for the constant harvesting model in Sec.3.3, so we leave out the complicated expressions. We only present the conclusions that the expectation of fish populations will be maximized at $T$ if we harvest $Q_{i}(i=1, \cdots, n)$ fish when their population grows to $N_{i}^{M}(i=1, \cdots, n)$ which are maximum points of the expectation of the fish population $\mathbb{E}[\ln N(T)]$ or minimum points of the expectation of the pest population.

To confirm analytical solutions in this subsection, as shown in Fig (5), we simulated a fish population 50 times and took the average. Fig (5)(a) shows that the expectation of the fish population reaches its maximal level when we conduct harvesting activities at the optimal thresholds $N_{1}^{M}$ and $N_{2}^{M}$ with different catch quotas $Q_{1}$ and $Q_{2}$. Fig (5)(b-d) shows the case of three-, four- and five-pulse impulsive non-constant harvesting, respectively.

In Fig (6)(a) and $\operatorname{Fig}(7)(a)$, the curve of a fish population with non-constant harvesting is simulated 30 times, 15 of which are the results of harvesting at optimal thresholds 
$N_{1}^{M}$ and $N_{2}^{M}$, and the rest are the results of harvesting at $N_{1}^{\prime}\left(>N_{1}^{M}\right)$ and $N_{2}^{\prime}\left(>N_{2}^{M}\right)$ in $\operatorname{Fig}(6)(\mathrm{a})$, while in $\operatorname{Fig}(7)(\mathrm{a})$ the remaining 15 curves are the results of harvesting at $N_{1}^{\prime \prime}\left(<N_{1}^{M}\right)$ and $N_{2}^{\prime \prime}\left(<N_{2}^{M}\right)$. Fig (6)(b-d) and Fig (7)(b-d) show the case of three-, fourand five-pulse impulsive non-constant harvesting, respectively.

\section{Estimation of fishing times}

In reality, what we want to know is when the density of the fish population reaches the optimal threshold $N_{i}$ at which the expectation of the fish population at the end of the season could be maximal, and this is more convenient for fishery resources management. However, it is challenging to find the exact time $\tau_{i}$ for a stochastic differential equation. Therefore, to address this we turn to estimate the range of the optimal time $\tau_{i}$ in this section.

\subsection{Estimation of fishing times}

For the one impulsive model, we have the following main results for the estimation of the optimal time $\tau_{i}$.

Theorem 4.1. Suppose $\tau:=\inf \left\{t>0: N(t)=N_{1}\right\}$ is a random time, and $r>\sigma^{2} / 2$, then the estimation of $\mathbb{E}[\tau]$ satisfies the inequality

$$
\frac{1}{r-\frac{1}{2} \sigma^{2}} \ln \frac{N_{1}}{N_{0}}<\mathbb{E}[\tau]<\frac{1}{r-\frac{1}{2} \sigma^{2}}\left(\ln \frac{N_{1}}{N_{0}}+\frac{N_{1}-N_{0}}{K}+\frac{(l-1) Q}{K}\right),
$$

where $l$ is a given positive integer.

Proof. Integrating the following equation

$$
\mathrm{d} N(t)=r N(t)\left(1-\frac{N(t)}{K}\right) \mathrm{d} t+\sigma N(t) \mathrm{d} B(t), N(0)=N_{0}
$$

yields

$$
N(t)=N_{0} e^{\left(r-\frac{1}{2} \sigma^{2}\right) t-\frac{r}{K} \int_{0}^{t} N(s) d s+\sigma B(t)},
$$

which indicates that we have the following inequality

$$
N(t)<N_{0} e^{\left(r-\frac{1}{2} \sigma^{2}\right) t+\sigma B(t)} \triangleq X(t)
$$

Note that $X(t)$ can be regarded as the solution of the following stochastic Malthusian model:

$$
\mathrm{d} X(t)=r X(t) \mathrm{d} t+\sigma X(t) \mathrm{d} B(t), \quad X(0)=N_{0} .
$$


It follows from (61) that we have

$$
N(t)>N_{0} e^{\left(r-\frac{1}{2} \sigma^{2}\right) t-\frac{r}{K} \int_{0}^{t} X(s) d s+\sigma B(t)} \triangleq Y(t) .
$$

Similarly, $Y(t)$ is the solution of the following stochastic model:

$$
\mathrm{d} Y(t)=r\left(1-\frac{X(t)}{K}\right) Y(t) \mathrm{d} t+\sigma Y(t) \mathrm{d} B(t), \quad Y(0)=N_{0}
$$

According to the estimation $Y(t)<N(t)<X(t)$, the random times $\tau_{m}:=\inf \left\{t>0: X(t)=N_{1}\right\}$ and $\tau_{M}:=\inf \left\{t>0: Y(t)=N_{1}\right\}$ must satisfy $\tau_{m}<\tau<\tau_{M}$.

(1) Using the Itô formula and taking the expectations in Eq.(62), we obtain

$$
\mathbb{E}\left[\ln X\left(\tau_{m}\right)\right]=\mathbb{E}\left[\ln N_{0}\right]+\left(r-\frac{1}{2} \sigma^{2}\right) \mathbb{E}\left[\tau_{m}\right]=\ln N_{1},
$$

which indicates that the lower boundary satisfies $\mathbb{E}\left[\tau_{m}\right]=1 /\left(r-0.5 \sigma^{2}\right) \ln \left(N_{1} / N_{0}\right)$.

(2) Now we estimate the upper boundary of the optimal time $\tau_{M}$. Integrating Eq.(62) on $\left[0, \tau_{m}\right]$ gives

$$
X\left(\tau_{m}\right)=N_{0}+\int_{0}^{\tau_{m}} r X(t) d t+\int_{0}^{\tau_{m}} \sigma X(t) d B(t) .
$$

Taking expectations, we have

$$
\mathbb{E}\left[X\left(\tau_{m}\right)\right]=N_{0}+\mathbb{E}\left[\int_{0}^{\tau_{m}} r X(t) d t\right]=N_{1}
$$

On the other hand, the expectations of $\ln Y\left(\tau_{M}\right)$ can be written as

$$
\mathbb{E}\left[\ln Y\left(\tau_{M}\right)\right]=\ln N_{0}+\left(r-\frac{1}{2} \sigma^{2}\right) \mathbb{E}\left[\tau_{M}\right]-\mathbb{E}\left[\frac{r}{K} \int_{0}^{\tau_{M}} X(t) d t\right]=\ln N_{1} .
$$

Substituting Eq.(67) into Eq.(68), we have

$$
\mathbb{E}\left[\tau_{M}\right]=\frac{1}{r-\frac{1}{2} \sigma^{2}}\left(\ln \frac{N_{1}}{N_{0}}+\frac{N_{1}-N_{0}}{K}+\mathbb{E}\left[\frac{r}{K} \int_{\tau_{m}}^{\tau_{M}} X(t) d t\right]\right) .
$$

Note that there must exist a positive integer $l$ such that the random times $\tau_{m}^{(2)}:=$ inf $\left\{t>\tau_{m}: X(t)=N_{1}\right\}$ and $\tau_{m}^{(j)}:=\inf \left\{t>\tau_{m}^{(j-1)}: X(t)=N_{1}\right\}$ for $j=3, \cdots, l$, as shown in $\operatorname{Fig}(8)$. Moreover, we have

$$
\mathbb{E}\left[\int_{\tau_{m}}^{\tau_{M}} r X(t) d t\right] \leqslant \mathbb{E}\left[\int_{\tau_{m}}^{\tau_{m}^{(2)}} r X(t) d t+\sum_{j=3}^{l} \int_{\tau_{m}^{(j-1)}}^{\tau_{m}^{(j)}} r X(t) d t\right]=(l-1) Q .
$$

All these results confirm that

$$
\mathbb{E}\left[\tau_{M}\right] \leqslant \frac{1}{r-\frac{1}{2} \sigma^{2}}\left(\ln \frac{N_{1}}{N_{0}}+\frac{N_{1}-N_{0}}{K}+\frac{(l-1) Q}{K}\right) .
$$


Hence, the random time $\tau$ satisfies the following inequality $\mathbb{E}\left[\tau_{m}\right]<\mathbb{E}[\tau]<\mathbb{E}\left[\tau_{M}\right]<T$. This completes the proof.

As an example, if we fixed all parameter values as those shown in $\operatorname{Fig}(8)$, from which we can see that the existence of the positive integer $l$ ensures that the optimal time $\tau_{M}$ lies in the estimation of the lower and upper boundaries. By employing similar methods, we can prove the following two results:

Corollary 4.1. In the multi-impulsive model, the optimal fishing times $\tau_{i}$ for $i=1, \cdots, n$ can also be estimated as follows:

$$
\begin{gathered}
\frac{1}{r-\frac{1}{2} \sigma^{2}} \ln \left(\prod_{k=1}^{i} \frac{N_{k}}{N_{k-1}^{+}}\right)<\mathbb{E}\left[\tau_{i}\right] \\
<\frac{1}{r-\frac{1}{2} \sigma^{2}}\left(\ln \left(\prod_{k=1}^{i} \frac{N_{k}}{N_{k-1}^{+}}\right)+\frac{N_{i}-N_{0}+(i-1) Q}{K}+\frac{(l-1) Q i}{K}\right)<T,
\end{gathered}
$$

here $N_{0}^{+}=N_{0}$.

Corollary 4.2. In the multi-impulsive model with different fishing quotas, the optimal fishing times $\tau_{i}$ for $i=1, \cdots, n$ can also be estimated as follows:

$$
\begin{gathered}
\frac{1}{r-\frac{1}{2} \sigma^{2}} \ln \left(\prod_{k=1}^{i} \frac{N_{k}}{N_{k-1}-Q_{k-1}}\right)<\mathbb{E}\left[\tau_{i}\right] \\
<\frac{1}{r-\frac{1}{2} \sigma^{2}}\left(\ln \left(\prod_{k=1}^{i} \frac{N_{k}}{N_{k-1}-Q_{k-1}}\right)+\frac{N_{i}-N_{0}+\sum_{k=1}^{i-1} Q_{k}}{K}+\frac{(l-1) \sum_{k=1}^{i} Q_{k}}{K}\right)<T,
\end{gathered}
$$

where $Q_{0}=0$.

\subsection{Numerical realization and implication}

We take the two-pulse constant fishing model as an example to examine the impact of parameter $Q$ with fixed $r=2, K=200$ and noise intensity $\sigma=0.4$. It is observed that if the harvest yield $Q$ is greater than or equal to 35, the discriminant of quadratic Eq.(31) is negative and there exists a pair of virtual roots which means that we cannot find an optimal fishing threshold $N_{1}$ to maximize fish populations when $N_{1}>2 Q$. On the other hand, a small decrease of $Q$ to 34 enables us to solve the optimal threshold which is 92 . Besides, for another occasion with $N_{1}<2 Q$, optimal thresholds always exist. It is worth determining which of these two optimal thresholds is better when $Q$ is less than or equal to 34 as shown in Fig(9). The conclusion is that we can get higher between season fish populations if harvesting activities take place at $N_{1}=97.85(>2 Q)$ when $Q$ is equal to 20 . 
Table 1: $\mathbb{E}\left[N_{1}\right]$ and $\mathbb{E}[\tau]$ varied as the noise intensity is changed. Parameter values are $N_{0}=10, K=200, r=2, Q=40, l=6$.

\begin{tabular}{lcc}
$\sigma$ & $\mathbb{E}\left[N_{1}\right]$ & $\mathbb{E}[\tau]$ \\
\hline 0.7 & 84.49 & {$[1.2160,1.6419]$} \\
0.5 & 103.57 & {$[1.2468,1.6963]$} \\
0.37 & 111.23 & {$[1.2472,1.7034]$} \\
0.2 & 117.48 & {$[1.2443,1.7051]$} \\
0.09 & 119.49 & {$[1.2428,1.7050]$} \\
0.006 & 119.998 & {$[1.2425,1.7050]$} \\
0.0006 & 119.99998 & {$[1.2425,1.7050]$} \\
\hline
\end{tabular}

Table 2: Optimal harvesting time in the deterministic and stochastic models. $\tau_{1}^{M}$ and $\mathbb{E}[\tau]$, respectively, represent the optimal harvesting time in the deterministic model and in the corresponding stochastic model. Parameter values are $N_{0}=10, K=200, r=2$, $\sigma=0.4$.

\begin{tabular}{cccccc}
$Q$ & $l$ & $\mathrm{~K}$ & $\frac{Q}{K}$ & $\tau_{1}^{M}$ & $\mathbb{E}[\tau]$ \\
\hline 15 & 6 & 200 & 0.075 & 1.5474 & {$[1.1929,1.6194]$} \\
20 & 5 & 200 & 0.1 & 1.5726 & {$[1.2045,1.6498]$} \\
30 & 4 & 200 & 0.15 & 1.6234 & {$[1.2267,1.7095]$} \\
40 & 3 & 200 & 0.2 & 1.6750 & {$[1.2475,1.7155]$} \\
45 & 3 & 200 & 0.225 & 1.7011 & {$[1,2574,1.7569]$} \\
\hline
\end{tabular}

In order to check how changing the noise intensity $\sigma$ affects the fish populations achieving a maximum, we consider the single-pulse constant harvesting model as an example. As shown in Tab.(1), the decrease in the noise intensity is found to reduce the threshold $N_{1}$, while the estimated optimal time varies a little. Moreover, it is interesting that a further decrease in $\sigma$ gets it to correspond with the conclusion of the deterministic model. Influences of single-pulse constant harvesting system parameters for different values of $\sigma$ are shown in Fig(10).

Optimal fishing time in the single-pulse constant harvesting deterministic model and the stochastic model for different $Q$ and $l$ with fixed $K$ are shown in Tab.(2). It is obvious that estimation of the optimal fishing time when the fish population reaches the optimal threshold is influenced by the factor $Q / K$. The optimal fishing time of the deterministic model is close to the right-hand end of the estimated interval for each $l$. For further clarification, we present the 20 curves of fish population when impulsive harvesting takes place at $N_{1}$ which is 109.7 as shown in Fig(11). All of the times when the simulated trajectories reach 109.7 are included in the estimated interval. 


\section{Conclusion}

In this paper we have considered a simple logistic impulsive model for the growth of a fish population under the influence of Gaussian white noise. An analytical technique was developed to investigate whether there exists an optimal harvesting time (or times) to maximize the fish population at the end of a harvest season. At first, the method of solving an extreme point was used to obtain the optimal thresholds in a single-impulse harvesting model, followed by a multi-impulse constant harvesting quota model as well as a multi-impulse non-constant quota model. Then we put forward a theory of estimating the time (or times) that will be useful in practice. Finally, theoretical predictions are verified by numerical calculations. The numerical results not only demonstrate the effectiveness of the proposed approach but also show the effects of noise intensity. Remarkably, the experimental results from the stochastic model are consistent with those of the deterministic model.

This proposed procedure can also be applied to deal with integrated pest management, when we focus on whether a pest population will reach a minimum at the end of a planting season. Based on the previous approach, we should work out the minimal value and then take advantage of the theorem of time estimation to find optimal times to apply pesticides. We emphasize that the modeling methods and analytical techniques could be applied in more sophistication cases including integrated pest management strategies, which have been extensively studied (Tang et al., 2012, 2015b,a).

Our results can be significant for understanding the exploitation of other renewable resources and for the design of management strategies. Due to the involvement of noise in a wide variety of fields we hope that our study will be applicable to solving some problems in many different disciplines.

\section{Acknowledgements}

This work was supported by the National Natural Science Foundation of China (NSFCs, 61772017, 11631012), and by the Fundamental Research Funds for the Central Universities GK201701001.

\section{References}

Anderson, L. G. (2004). The Economics of fisheries management. The Blackburn Press, New Jersey.

Arnold, L., Horsthemke, W., and Stucki, J. W. (1979). The influence of external real and white noise on the lotka-volterra model. Biometrical J., 21(5):451-471.

Bandyopadhyay, M., Saha, T., and Pal, R. (2008). Deterministic and stochastic analysis of a delayed allelopathic phytoplankton model within fluctuating environment. Nonlinear Anal. Hybrid Syst., 2(3):958-970.

Cai, Y., Kang, Y., and Wang, W. (2017). A stochastic SIRS epidemic model with nonlinear incidence rate. Appl. Math. Comput., 305:221-240. 
Campillo, F., Joannides, M., and Larramendy-Valverde, I. (2011). Stochastic modeling of the chemostat. Ecol. Modell, 222:2676-2689.

Campillo, F. and Lobry, C. (2012). Effect of population size in a predator-prey model. Ecol. Modell, 246:1-10.

Cao, Y. and Tang, S. (2016). The threshold of pest control in a stochastic malthus model. J. Biomath., 31(1):83-92.

Caraballo, T. (1990). Asymptotic exponential stability of stochastic partial differential equations with delay. Stoch. Anal. Appl., 17(5):743-763.

Castro-santis, R., Córdova-lepe, F., and Chambio, W. (2016). An impulsive fishery model with environmental stochasticity. feasibility. Math. Biosci., 277:71-76.

Clark, C. (1976). Mathematical Bioeconomics: The Optimal Management of Renewable Resources. John Wiley, New York.

Dong, L., Chen, L., and Sun, L. (2007). Optimal harvesting policies for periodic gompertz systems. Nonlinear Anal. RWA, 8:572-578.

Hassani, H., Dionisio, A., and Ghodsi, M. (2010). The effect of noise reduction in measuring the linear and nonlinear dependency of financial markets. Nonlinear Anal. RWA, 11(1):492-502.

Imhof, L. and Walcher, S. (2005). Exclusion and persistence in deterministic and stochastic chemostat models. J. Diff. Equ., 217:26-53.

Ji, C., Jiang, D., Shi, N., and O'Regan, D. (2007). Existence, uniqueness, stochastic persistence and global stability of positive solutions of the logistic equation with random perturbation. Math. Method Appl. Sci., 30(1):77-89.

Kloeden, P. E. and Pearson, R. A. (1992). Numerical solution of stochastic differential equations. Springer Berlin Heidelberg.

Liu, K. and Mao, X. (1998). Exponential stability of non-linear stochastic evolution equations. Stoch. Proc. Appl., 78(2):173-193.

Liu, M. and Wang, K. (2012). On a stochastic logistic equation with impulsive perturbations. Comput. Math. Appl., 63:871-886.

Lu, C. and Ding, X. (2014). Persistence and extinction of a stochastic logistic model with delays and impulsive perturbation. Acta Math. Sci., 34(5):1551-1570.

Mao, X. (2007). Stochastic differential equations and applications. second ed. Horwood Publishing Limited, Chichester, London.

Mao, X., Sabanis, S., and Renshaw, E. (2003). Asymptotic behaviour of the stochastic lotka volterra model. J. Math. Anal. Appl., 287(1):141-156. 
Nundloll, S., Mailleret, L., and Grognard, F. (2010). Two models of interfering predators in impulsive biological control. J. Biol. Dyn., 4:102-114.

Punt, A. and Hilborn, R. (1997). Fisheries stock assessment and decision analysis: the bayesian approach. Rev. Fish Biol. Fisher., 7(1):35-63.

Sakthivel, R. and Luo, J. (2009). Asymptotic stability of nonlinear impulsive stochastic differential equations. Stat. Probabil. Lett., 79(9):1219-1223.

Schaffer, W. M., Ellner, S., and Kot, M. (1986). Effects of noise on some dynamical models in ecology. J.Math. Biol., 24(5):479-523.

Szuwalski, C., Castrejon, M., Dan, O., and Chasco, B. (2016). An integrated stock assessment for red spiny lobster ( panulirus penicillatus ) from the galapagos marine reserve. Fish. Res., 177:82-94.

Tang, S. and Cheke, R. (2008). Models for integrated pest control and their biological implications. Math. Biosci., 215:115-125.

Tang, S. and Chen, L. (2004). The effect of seasonal harvesting on stage-structured population models. J. Math. Biol., 48(4):357-374.

Tang, S., Liang, J., Tan, Y., and Cheke, R. (2013). Threshold conditions for integrated pest management models with pesticides that have residual effects. J. Math. Biol., 66(1-2):1-35.

Tang, S., Liang, J., Xiao, Y., and Cheke, R. (2012). Sliding bifurcations of filippov two stage pest control models with economic thresholds. SIAM J. Appl. Math., 72(4):10611080 .

Tang, S., Pang, W., Cheke, R., and Wu, J. (2015a). Global dynamics of a state-dependent feedback control system. Adv. Diff. Equ., 322(1):1-70.

Tang, S., Tang, B., Wang, A., and Xiao, Y. (2015b). Holling ii predator-prey impulsive semi-dynamic model with complex poincare map. Nonlin. Dyn., 81(1):1-22.

Tang, S., Xiao, Y., Chen, L., and Cheke, R. (2005). Integrated pest management models and their dynamical behaviour. B. Math. Biol., 67(1):115-135.

Terry, A. J. and Gourley, S. A. (2010). Perverse consequences of infrequently culling a pest. Bull. Math. Biol., 72:1666-1695.

Wang, W., Cai, Y., Li, J., and Gui, Z. (2017). Periodic behavior in a FIV model with seasonality as well as environment fluctuations. J. Franklin Inst., 354:7410-7428.

Xiao, Y., Cheng, D., and Qin, H. (2006). Optimal impulsive control in periodic ecosystem. Syst. Control Lett., 55(7):558-565.

Xue, Y., Tang, S., and Liang, J. (2012). Optimal timing of interventions in fishery resource and pest management. Nonlinear Anal. RWA, 13(4):1630-1646. 
Yeh, J. (1995). Martingales and stochastic analysis. World Scientific, Singapore.

Zhang, X., Shuai, Z., and Wang, K. (2003). Optimal impulsive harvesting policy for single population. Nonlinear Anal. RWA, 4:639-651.

Zhao, L., Zhang, Q., and Yang, Q. (2003). Optimal impulsive harvesting for fish populations. J. Syst, Sci. Complex, 16:466-474.

Zhao, T. and Tang, S. (2011). Impulsive harvesting and by-catch mortality for the theta logistic model. Appl. Math. Comput., 217:9412-9423.

Zou, X., Fan, D., and Wang, K. (2013). Stationary distribution and stochastic hopf bifurcation for a predator-prey system with noises. Discrete Cont. Dyn.B, 18(5):15071519 . 
Figure Legends
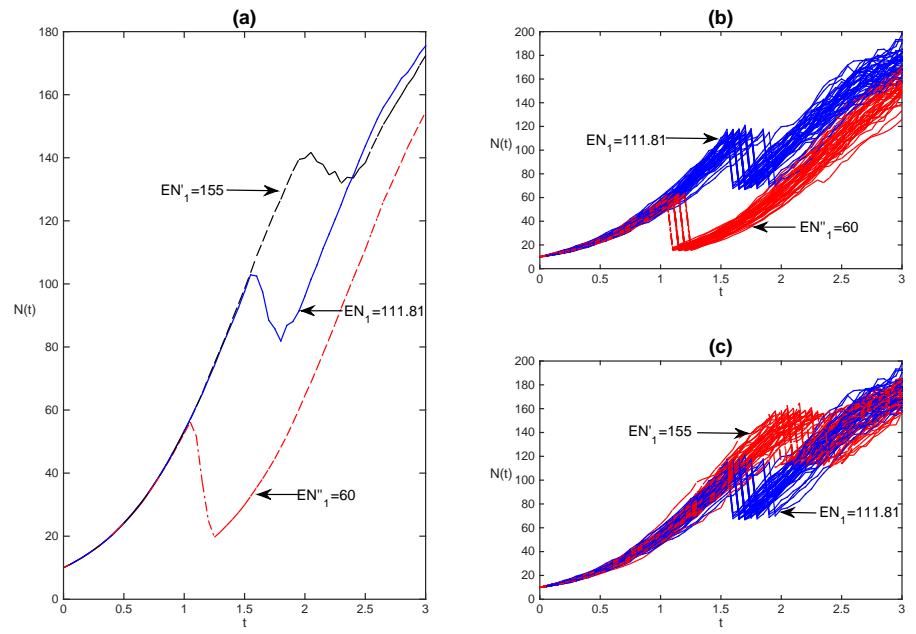

Figure 1: Each curve in (a) is the average of 40 simulations of a stochastically fluctuating fish population with constant harvesting. The solid blue line denotes the fish population arriving at its maximal level at the end of the harvesting season if we harvest fish at the optimal threshold level $N_{1}=N_{1}^{M}$. The dotted black line and the dashed-dotted red line are set as control groups. Corresponding non-average curves are shown in (b) and (c). Parameter values are $N_{0}=10, K=200, r=2, \sigma=0.4, Q=45$.
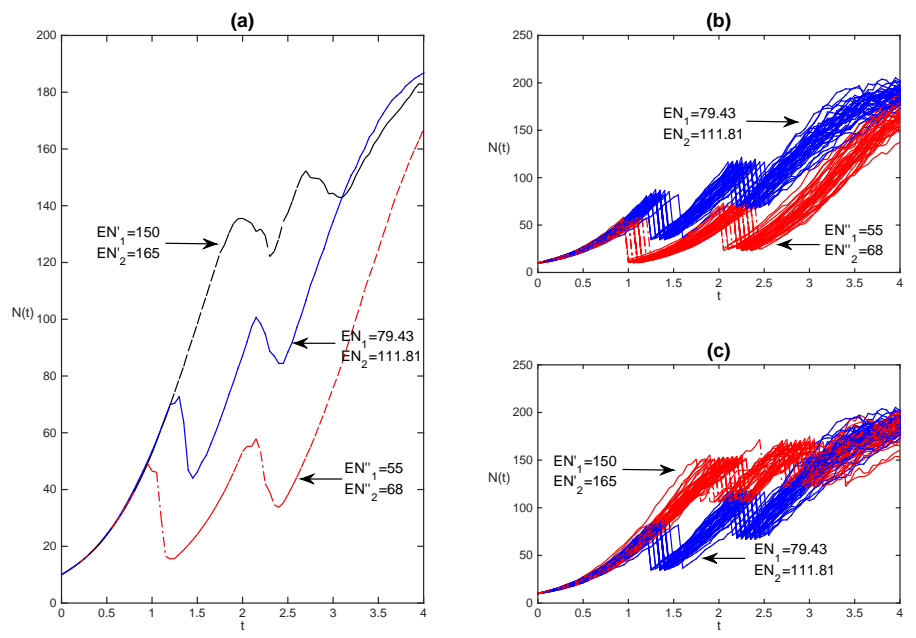

Figure 2: Each curve in (a) is the average of 40 simulations of a stochastically fluctuating fish population with constant harvesting. The solid blue line denotes the fish population arriving at its maximal level at the end of the harvesting season if we harvest fish at the optimal threshold $\left(N_{1}, N_{2}\right)=\left(N_{1}^{M}, N_{2}^{M}\right)$. The dotted black line and the dashed-dotted red line are set as control groups. Corresponding non-average curves are shown in (b) and (c). Parameter values are $N_{0}=10, K=200, r=2, \sigma=0.4, Q=45$. 

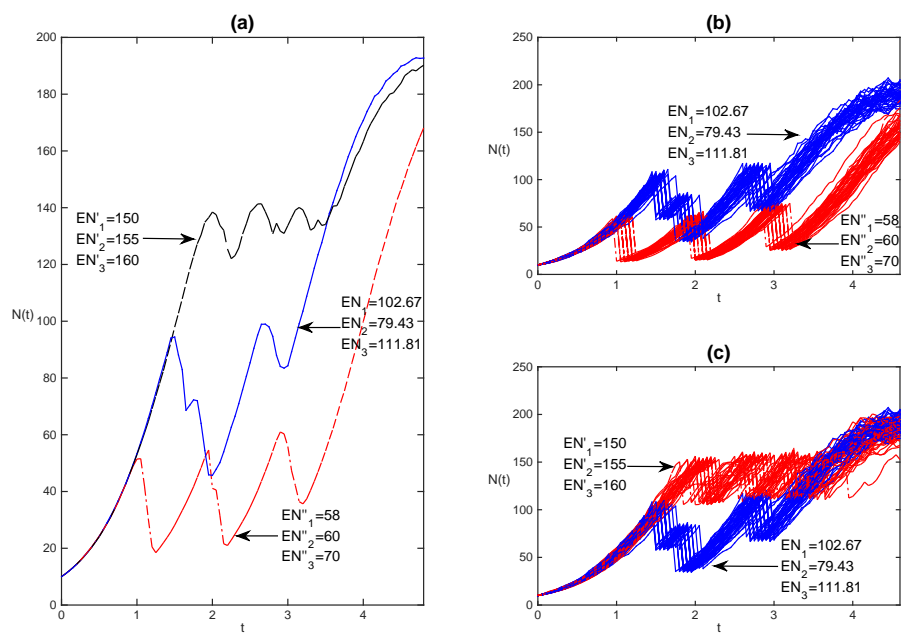

Figure 3: Each curve in (a) is the average of 40 simulations of a stochastically fluctuating fish population with constant harvesting. The solid blue line denotes the fish population arriving at its maximal level at the end of the harvesting season if we harvest fish at the optimal thresholds $N_{i}=N_{i}^{M}$ for $i=1,2,3$. The dotted black line and the dashed-dotted red line are set as control groups. Corresponding non-average curves are shown in (b) and (c). Parameter values are $N_{0}=10, K=200, r=2, \sigma=0.4, Q=45$.
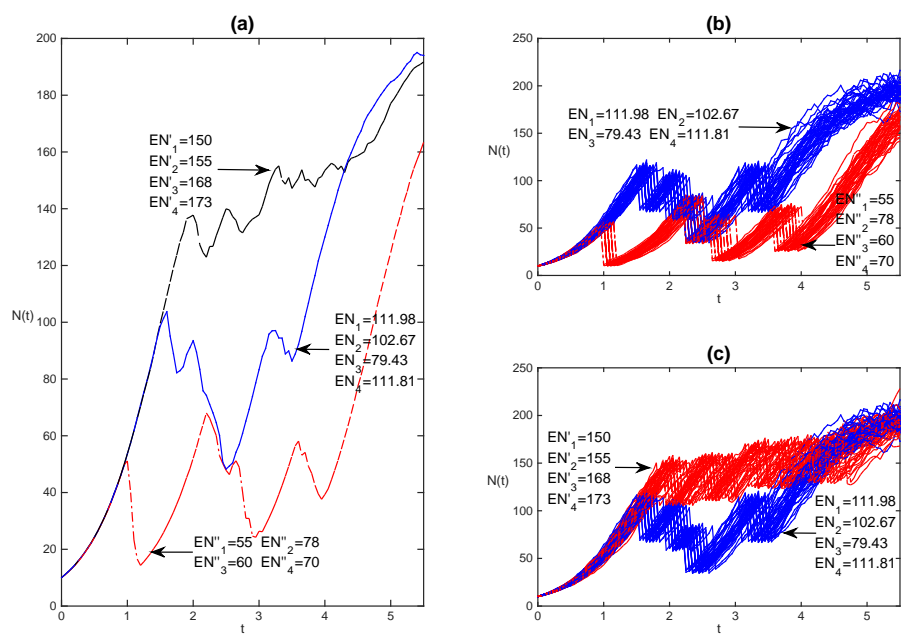

Figure 4: Each curve in (a) is the average of 40 simulations of a stochastically fluctuating fish population with constant harvesting. The solid blue line denotes the fish population arriving at its maximal level at the end of the harvesting season if we harvest fish at the optimal thresholds $N_{i}=N_{i}^{M}$ for $i=1, \cdots, 4$. The dotted black line and the dashed-dotted red line are set as control groups. Corresponding non-average curves are shown in (b) and (c). Parameter values are $N_{0}=10, K=200, r=2, \sigma=0.4, Q=45$. 

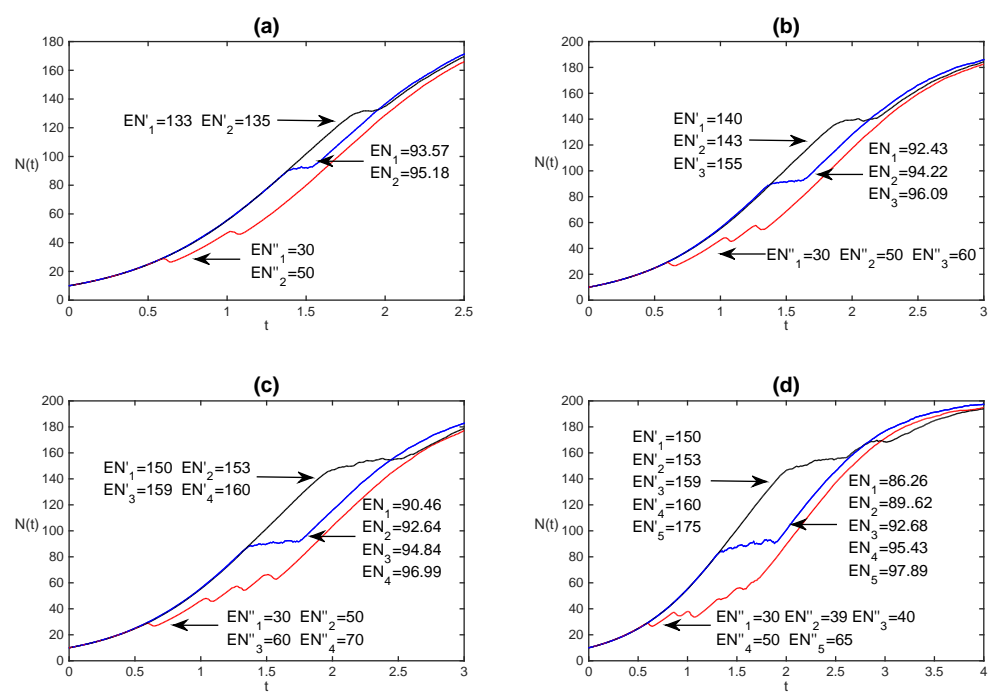

Figure 5: Each curve is the average of 50 simulations of a stochastically fluctuating fish population with non-constant harvesting. The blue line denotes the fish population's arrival at its maximal level at the end of the harvesting season if we harvest fish at the optimal thresholds $N_{i}=N_{i}^{M}$. The black line and the red line are set as control groups. Parameter values are $N_{0}=10, K=200, r=2, \sigma=0.4, Q_{1}=5, Q_{2}=7, Q_{3}=9$, $Q_{4}=11, Q_{5}=13$.
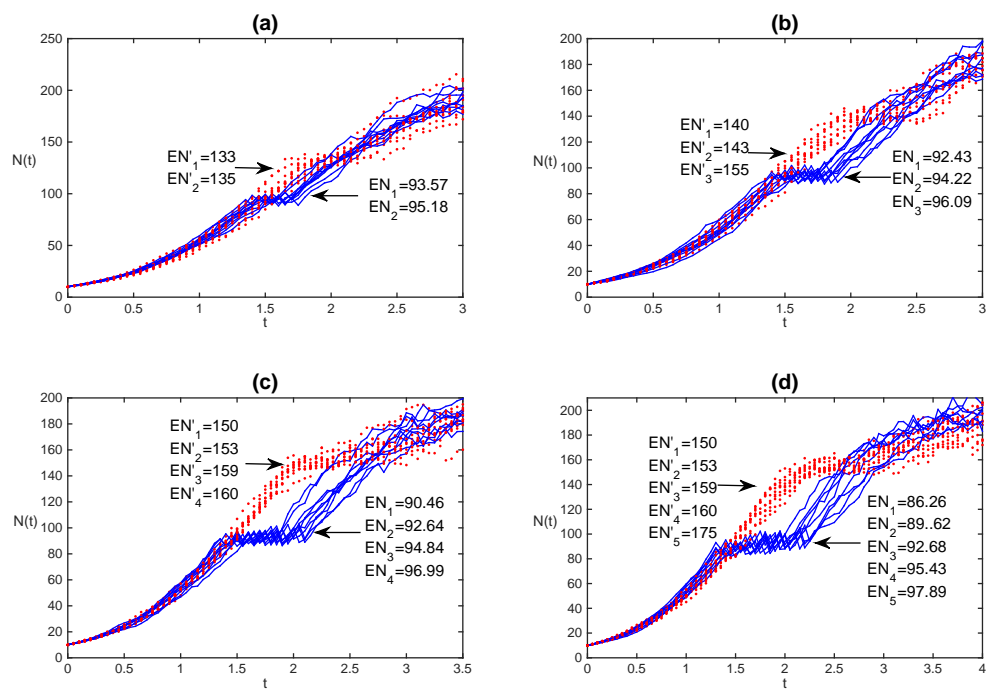

Figure 6: Simulated trajectories showing the comparisons between harvesting at optimal times and at other times in the multi-pulse non-constant harvesting model. The solid line represents optimal harvesting taking place and the dashed line denotes non-optimal impulsive harvesting. Parameter values are the same as those in Fig.(5)(a) Single-pulse harvesting; (b) Two-pulse harvesting; (c) Three-pulse harvesting; (d) Four-pulse harvesting. 
(a)

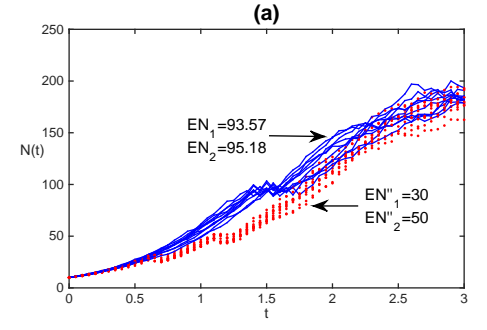

(c)

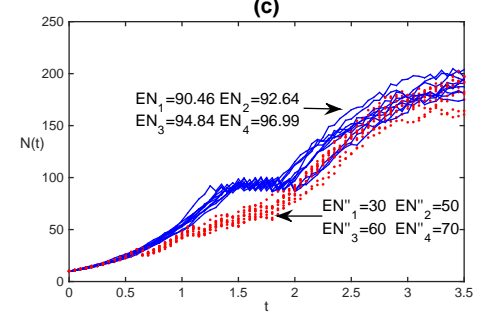

(b)

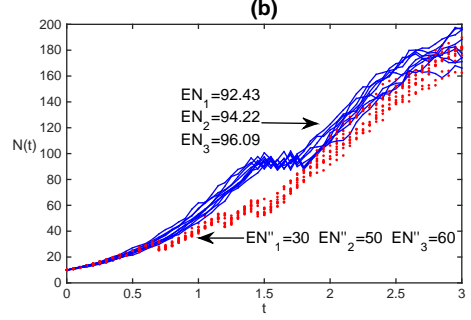

(d)

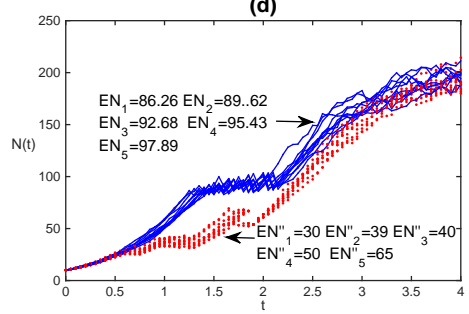

Figure 7: Simulated trajectories showing the comparisons between harvesting at optimal times and at other times in the multi-pulse non-constant harvesting model. The solid line represents optimal harvesting taking place and the dashed line denotes non-optimal impulsive harvesting. Parameter values are the same as those in Fig.(5)(a) Single-pulse harvesting; (b) Two-pulse harvesting; (c) Three-pulse harvesting; (d) Four-pulse harvesting.
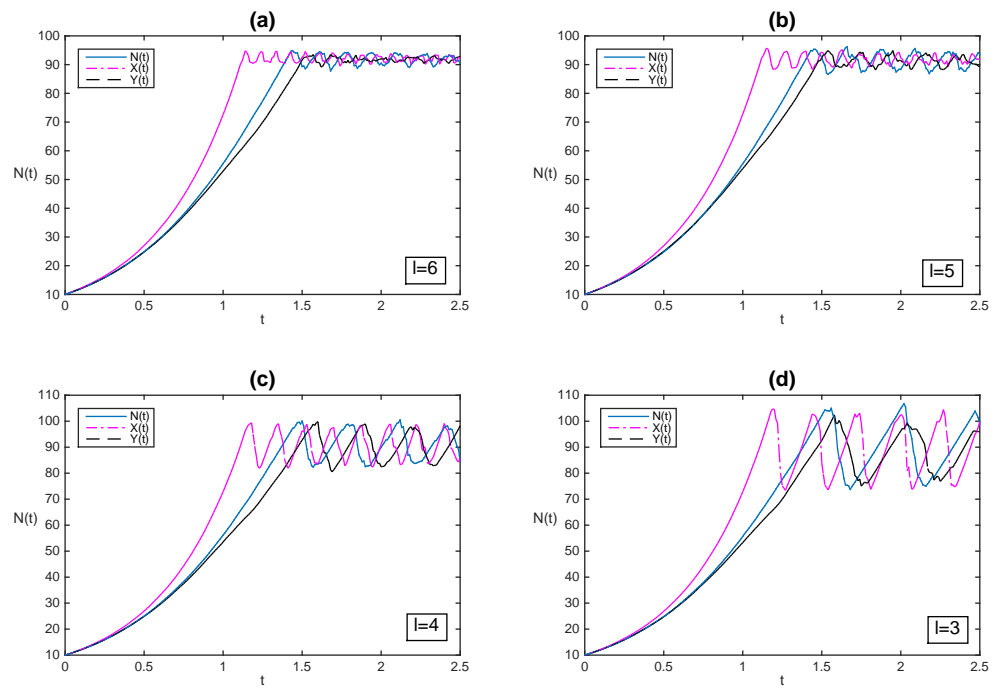

Figure 8: Illustration of the existence of positive integer $l$ such that $\tau_{m}^{(l-1)}<\tau_{M}<\tau_{m}^{(l)}$ and each curve is the average of 40 simulations of stochastically fluctuating fish population with constant harvesting $Q$. Parameter values are $N_{0}=10, K=200, r=2, \sigma=0.4$, (a) $Q=15, N_{1}=98.78$; (b) $Q=20, N_{1}=101.01$; (c) $Q=30, N_{1}=105.41$; (d) $Q=45$, $N_{1}=111.81$. The time step is $d t=0.01$. 

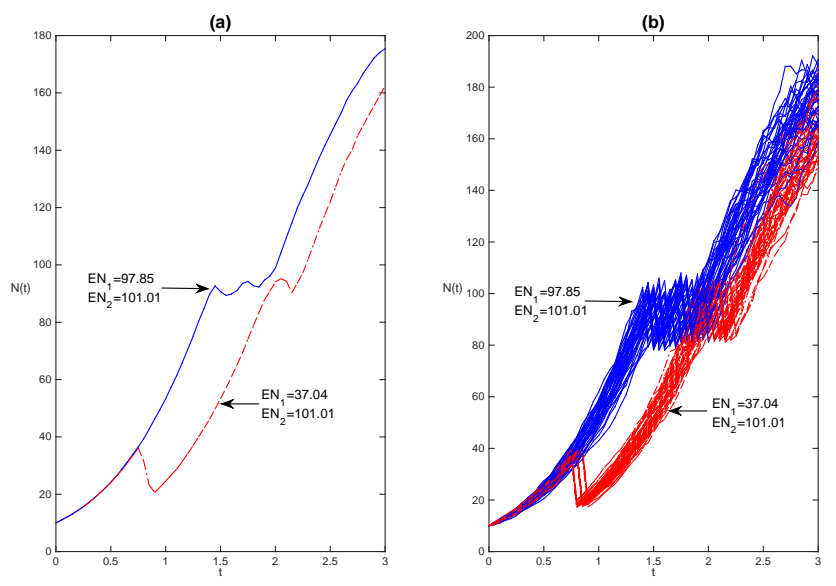

Figure 9: Trajectories with and without the average of the fish population if we harvest fish at the optimal thresholds. The solid blue lines are the cases of optimal impulsive harvesting taken place at $N_{1}>2 Q$. The dashed-dotted red lines denote impulsive harvesting activities implemented at $N_{1}<2 Q$. Parameter values are $N_{0}=10, K=200, r=2, Q=20$ and $\sigma=0.4$.
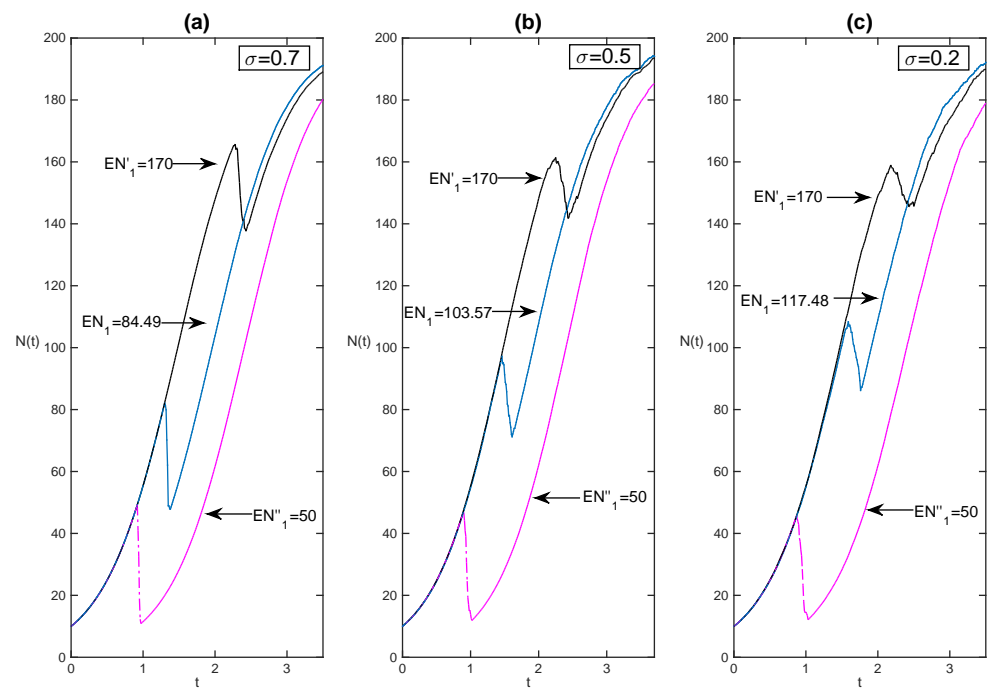

Figure 10: Trajectories of the single-pulse constant harvesting model for different noise intensities. The solid line denotes the fish population's arrival at its maximal level at the end of the harvesting season if we harvest fish at the optimal times; The dotted line and the dashed-dotted line are set as control groups. Parameter values are $N_{0}=10, K=200$, $r=2, Q=40$, (a) $\sigma=0.7$; (b) $\sigma=0.5$; (c) $\sigma=0.2$. 

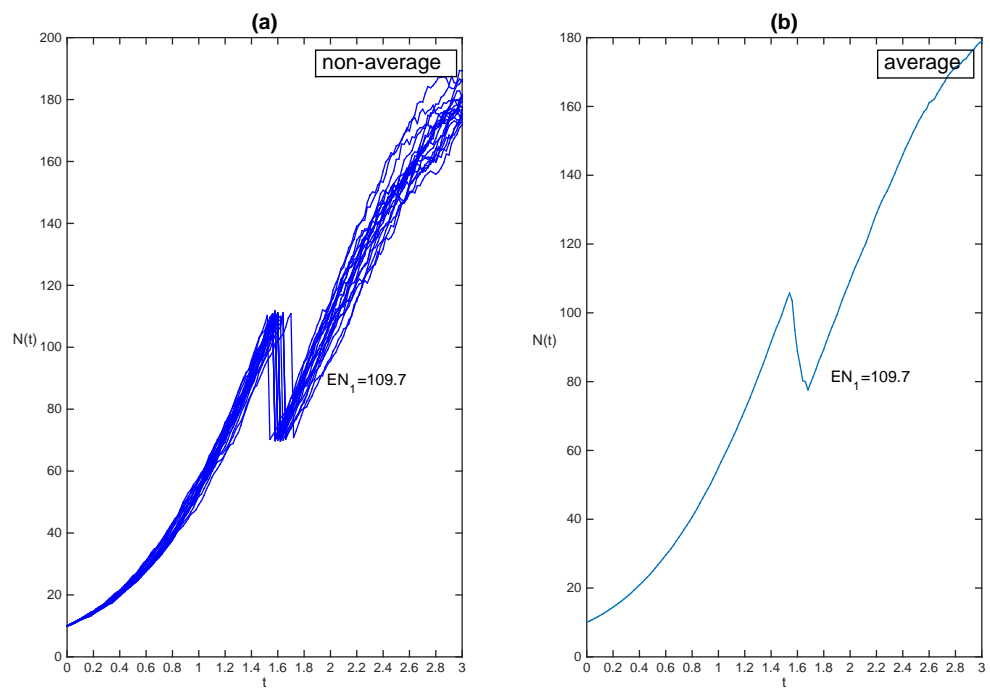

Figure 11: Trajectories of the single-pulse constant harvesting model. Twenty curves in (a) denote the fish population's arrival at its maximal level at the end of the harvesting season if we harvest fish at the optimal times. The average is shown in (b).Parameter values are $N_{0}=10, K=200, r=2, Q=40$ and $\sigma=0.4$. 